\title{
Ramp metering and freeway bottleneck capacity
}

\author{
Lei Zhang ${ }^{\mathrm{a}, 1}$, David Levinson ${ }^{\mathrm{b}, *}$ \\ a Department of Civil and Environmental Engineering, University of Maryland, 1173 Glenn Martin Hall, College Park, MD 20742, United States \\ ${ }^{\mathrm{b}}$ Department of Civil Engineering, University of Minnesota, 122 Civil Engineering Building, 500 Pillsbury Drive SE, Minneapolis, MN 55455, United States
}

\section{A R T I C L E I N F O}

\section{Article history:}

Received 20 January 2009

Received in revised form 8 January 2010

Accepted 16 January 2010

\section{Keywords:}

Ramp metering

Highway capacity

Active bottleneck

Queue discharge flow

Twin Cities ramp meter shut-off

\begin{abstract}
A B S T R A C T
This study aims to determine whether ramp meters increase the capacity of active freeway bottlenecks. The traffic flow characteristics at 27 active bottlenecks in the Twin Cities have been studied for seven weeks without ramp metering and seven weeks with ramp metering. A methodology for systematically identifying active freeway bottlenecks in a metropolitan area is proposed, which relies on two occupancy threshold values and is compared to an established diagnostic method - transformed cumulative count curves. A series of hypotheses regarding the relationships between ramp metering and the capacity of active bottlenecks are developed and tested against empirical traffic data. It is found that meters increase the bottleneck capacity by postponing and sometimes eliminating bottleneck activations, accommodating higher flows during the pre-queue transition period, and increasing queue discharge flow rates after breakdown. Results also suggest that flow drops after breakdown and the percentage flow drops at various bottlenecks follow a normal distribution. The implications of these findings on the design of efficient ramp control strategies, as well as future research directions, are discussed.
\end{abstract}

(c) 2010 Elsevier Ltd. All rights reserved.

\section{Introduction}

Ramp metering, since its debut in the early 1960s (May, 1964), has been widely deployed in many urban areas. Meters can reduce average freeway commuter delay by appropriately managing entrance ramp inflows. Other positive impacts of ramp metering, such as improved safety, more reliable travel time, reduced emission and fuel consumption, and high occupancy vehicle (HOV) priority, traffic diversion to under-utilized local streets, have also been reported in past studies (e.g. Zhang, 2007; Levinson and Zhang, 2006; Cambridge Systematics, 2001), but are often considered as secondary objectives even though their benefits may exceed the reduction in travel delay. When designing ramp metering algorithms, engineers and researchers usually focus on the improvement of freeway mainline capacity. However, the fundamental relationship between ramp metering and freeway capacity has not been adequately studied. The important question of whether ramp metering increases the capacity at freeway bottlenecks has not found a conclusive answer. A better understanding of this issue would generate information valuable to both researchers and practitioners, such as control threshold values (i.e. the optimal volume or density used as control objectives) in local or coordinated ramp metering algorithms.

Active bottlenecks are characterized by queue discharge flows not affected by traffic conditions further downstream (Daganzo, 1997). Several studies, by carefully examining traffic data before and after breakdown at active bottlenecks, reveal that maximum flow rates diminish after queues form upstream (Cassidy and Bertini, 1999; Hall and Agyemang-Duah, 1991; Banks, 1991a,b). The two-capacity hypothesis argues that metering can increase bottleneck capacity by preventing, or at

\footnotetext{
* Corresponding author. Tel.: +1 612625 6354; fax: +1 6126267750.

E-mail addresses: lei@umd.edu (L. Zhang), dlevinson@umn.edu (D. Levinson).

1 Tel.: +1 301405 2881; fax: +1 3014052585 .
} 
least postponing queue formation on the freeway mainline (Banks, 1990). Several simulation studies with simplified demand patterns on hypothetical networks provide evidence that ramp metering can increase bottleneck flows in a simulated environment (Persaud et al., 2001; Kotsialos et al., 2002). Whether it is also true in reality is not clear. The work of Cassidy and Rudjanakanoknad (2002) examines flow characteristics at one merging bottleneck with four days of metering-off and -on data. Their method with a focused local setup can provide detailed information for analysis of capacity-related issues at individual bottlenecks. It should be pointed out that some other studies suggest the two-capacity phenomenon does not provide a basis for ramp metering because the observed flow drop after breakdown seems insignificant (Newman, 1961; Persaud, 1986; Persaud and Hurdle, 1991).

The overall objective of this study is to determine whether ramp metering increases the capacity at freeway bottlenecks, and statistically test this hypothesis against a large empirical multi-bottleneck dataset. As suggested by Banks (1990), the most direct way to answer this question would be to experiment with metering rates, including turning the meters off. Another approach is to document flow processes carefully in the vicinity of bottlenecks to determine the possible effects of metering. Most previous studies adopted the second approach, probably due to the lack of traffic data under different metering status. The shortfall of this approach is that several important questions cannot be answered with metering-on or -off traffic data alone. For instance, these studies cannot test whether the queue discharge flow rates with metering are significantly higher than without metering. It is fortuitous for researchers that the Twin Cities ramp metering shut-off experiment occurred in 2000. The database constructed during the experiment allows us to examine more than $250 \mathrm{~km}$ of freeways for a long period of time with and without ramp metering. A detailed description of the background and the timeline of this shut down experiment can be found in previous studies (Levinson and Zhang, 2006; Zhang and Levinson, 2004a). Using this data set, we are able to statistically test several hypothesized relationships between ramp metering and freeway bottleneck capacity.

Before proceeding to the analysis, we would like to discuss several important concepts and terms regarding ramp metering which will be referred to later in this paper. Such a discussion also helps readers understand the value and limitations of this work. First, increased capacity at bottlenecks is not a necessary condition for ramp metering to reduce commuter delay. The real necessary condition with fixed freeway demand is that ramp metering shifts the system departure curve in a manner such that the area bounded by the departure and arrival curves in the queuing diagram is narrowed. In terms of terminology, we reserve output for the departure rate of the whole freeway system (mainline plus ramps), and capacity for homogenous freeway sections. We follow the capacity definition of Zhang and Levinson (2004b), which states capacity at a bottleneck is a weighted sum of its pre-queue transition flow rate and queue discharge flow rate. Ramp metering increases freeway bottleneck capacity if it increases both pre-queue transition flow and queue discharge flow rates. Even if the capacity at bottlenecks does not increase with metering (we refer to capacity increase at bottlenecks as a Type II capacity increase hereafter in the paper), the flow on mainline sections and exit ramps upstream of bottlenecks may increase because ramp meters to some extent prevent queue formation and in case a queue has already formed, limit its physical length, so that fewer exit ramps are blocked and trips with destination off-ramps upstream of the bottleneck are either not delayed or delayed less (a Type I capacity increase). Therefore, total output can still be improved even though nothing changes at bottlenecks. This benefit of ramp metering was recently expounded by Cassidy (2003). Therefore, reduced freeway travel time or delay does not necessarily provide evidence that ramp metering increases bottleneck capacity unless observed off-ramp flow increases are not sufficient by themselves to explain the travel time reduction. For the same reason, previous studies not explicitly considering active bottlenecks shed no light on the hypotheses tested in the present paper. It should also be noted that identifying the optimal control strategy is not the goal of this research, though some results from this empirical analysis may be used by future studies toward that goal. In that case, the impact of traffic waiting at meters must be considered.

Section 2 of this paper describes the study sites and traffic data in detail. Section 3 develops a series of statistically testable hypotheses about the impacts of ramp metering on bottleneck capacity. For the purpose of this research, an appropriate data analysis tool is required to help identify active bottlenecks, queue discharge flows and other related traffic characteristics. Section 4 illustrates the occupancy method with two threshold values used in this study, followed by results of hypothesis testing in Section 5. The final section concludes the study and discusses the implications of the findings on the design of effective ramp control strategies.

\section{Data and study sites}

Seventy-five percent of the Twin Cities metro area freeways are served by a traffic management system including cameras, inductive loop detectors, ramp meters, variable message signs, and incident management. The Minnesota Department of Transportation (MnDOT) installed the first ramp meter on I-35E in 1969 in the Twin Cities metropolitan area. The ramp metering system has since grown to include 440 ramp meters with 235 operating during the morning peak period and 285 operating during the afternoon peak period by the time a bill passed in the 2000 Minnesota Legislature requiring a shut down experiment to study the effectiveness of the system. The experiment provided 32 flow and occupancy data from nearly 4000 single loop detectors during a seven-week period (from the third week of October to the first week of December in 2000) without ramp metering. Although loop detector data for the metering-on scenario are available for all days after installation, only the corresponding seven weeks in 1999 were selected as the study period for the metering-on scenario for reasons explained later in this section. Raw traffic data (flow and occupancy) were extracted in 30-s intervals from MnDOT 
archived binary data files which are available to the public (TDRL, 2004). Video data collected by surveillance cameras were, however, not archived for privacy concerns. Since it is impossible to validate detector data with video information, a comprehensive detector error test was performed to ensure that data from corrupt detectors were not included in the analysis. Past studies (Chen and May, 1987; Jacobson et al., 1990) proposed various methods for identifying corrupt detectors, mentioned eight types of detector failures, and suggested corresponding test algorithms (missing data, zero volume and occupancy, zero volume and 100\% occupancy, constant non-zero volume and occupancy, zero volume but non-zero occupancy, non-zero volume but zero occupancy, practically impossible volume ( $>3600$ veh/ln/h) or occupancy ( $>90 \%$ ), bad flow conservation at adjacent detection stations (flow discrepancies $>500 \mathrm{veh} / \mathrm{lane} / 24 \mathrm{~h}$ )). These tests were performed on all raw traffic data used in this study and corrupt detectors were simply eliminated from the analysis. No efforts have been made to repair missing or suspicious data. More specifically, we wrote a Java program which reports detection malfunctioning information including start time, duration, and type of malfunctioning. If any type of detection error lasted for more than two consecutive intervals in which traffic data might be used for the subsequent capacity analysis, the corresponding detector and the detection station will be considered as corrupt for the whole peak period.

In 1999, all studied freeways were controlled by the Minnesota zonal metering algorithm, a real-time, coordinated strategy that limits inflows at predetermined groups of on-ramps upstream of bottlenecks in order to keep bottleneck flows strictly below some flow threshold values (MnDOT, 1998; Bogenberger and May, 1999). The threshold values are usually around $2220 \mathrm{veh} / \mathrm{lane} / \mathrm{h}$ based on historical data. Several occupancy threshold values are also established in the algorithm to detect queues. If the algorithm identifies that breakdown has already occurred and a queue is present (marked by high occupancy values upstream of the bottleneck), stricter metering rates will be applied in order to dissipate the mainline queue. This study focuses on the afternoon peak period (13:00-21:00). The earliest possible starting time and the latest possible ending time of ramp metering operation during afternoon peak periods are 14:30 and 19:30 respectively. The study has also been restricted to normal weekdays so that the driving population is relatively constant and familiar with the facilities. The Thanksgiving holidays are omitted.

Besides the metering status, weather conditions, demand fluctuation, accidents, and road maintenance activities all have significant impacts on measured flow and occupancy values, and can potentially bias hypothesis testing results. Therefore, we developed and implemented a data filter in order to appropriately control for those factors. Weather data were downloaded from National Oceanic and Atmospheric Administration hourly weather reports at seven Twin Cities observation stations. If the weather stations reported snow or more than one centimeter of rain during a peak period in a day, that day would be eliminated from the analysis of corresponding detector stations. If there were lane-blocking accidents or road construction activities in the vicinity of a detection station in a day, and the traffic data suggested an abnormal pattern, that day would also be eliminated from the analysis. The accident data was collected by the MnDOT Incident Management Program which includes the characteristics, starting time, and clearing time of all identified non-recurrent incidents. Seasonal demand fluctuations were taken into account by analyzing data during the corresponding seven weeks with and without ramp metering, which also ensures similar lighting conditions (sun angle, visibility, etc.) in both scenarios (Koshi et al., 1992 found a significant flow jump immediately following sunrise on a Japanese freeway, which suggests sunlight could be an important factor). Demand patterns may vary from 1999 to 2000 due to annual traffic growth. Ramp metering itself induces certain unique demand responses. Zhang and Levinson (2002) demonstrate that freeways in general carry more trips but fewer vehicle kilometers of travel without metering than with metering. Section 5 illustrates how we explicitly control for annual traffic growth. Finally, in order to minimize the impact of normal detection noise, 10-min moving averages of raw flow and occupancy data with 32 successive intervals were computed and used in the following analysis after experiments with several aggregation intervals.

The above data filtering process excluded a surprisingly high number of bottlenecks from the analysis primarily due to detection errors. Out of several-hundred candidate sites, only 27 active bottlenecks identified by a methodology described later in Section 4 survived the above selection criteria. All of them are recurrent bottlenecks where the number of breakdown occurrences during the metering-off period ranges from 8 to 74 . The location and geometric characteristics of these studied bottlenecks are summarized in Figs. 1 and 2 respectively. The sample has a good mix of various types of bottlenecks and some bottlenecks have multiple characteristics that may cause traffic breakdown. A bottleneck is considered as a weaving section if it is a short joint section $(<1 \mathrm{~km})$ of two major freeways (bottlenecks 9,12 , and 24$)$ or caused by weaving from both an on-ramp and an off-ramp (bottlenecks 1 and 10). Some bottlenecks are located near bridges with narrow shoulders or inside tunnels (bottlenecks 5, 19, 20, and 27). Some bottlenecks are located in freeway sections with visually identifiable horizontal curves (bottlenecks 12,16,17, and 21), or uphill grade along the direction of travel (bottleneck 15), or both (bottlenecks 20,23, 26, and 27). One bottleneck is caused by lane drop from three to two (bottleneck 25). If there is an on-ramp located no further than $1.6 \mathrm{~km}$ (one mile) upstream, a bottleneck is at least partially due to weaving from the on-ramp (bottlenecks $2-8,11,13-23$, and 25-27), even though the average hourly peak period volume at several upstream on-ramps is not very high ( $<250 \mathrm{veh} / \mathrm{h}$ at bottlenecks 5 and 16$)$. These categorization criteria tend to list all possible causes of traffic breakdown at each studied bottleneck, some of which might not be significant. However, there is no way to test the performance of those bottlenecks without one or more of the listed characteristics and to eliminate the insignificant factors.

The locations of data detection stations with respect to the bottlenecks are also shown in Fig. 2. Loop detectors are installed at about $0.8-\mathrm{km}$ (half-mile) intervals on Twin Cities freeways, and additional detection stations are installed in merging and diverging areas to ensure direct detection of traffic data in every freeway segment with uniform flow characteristics. 


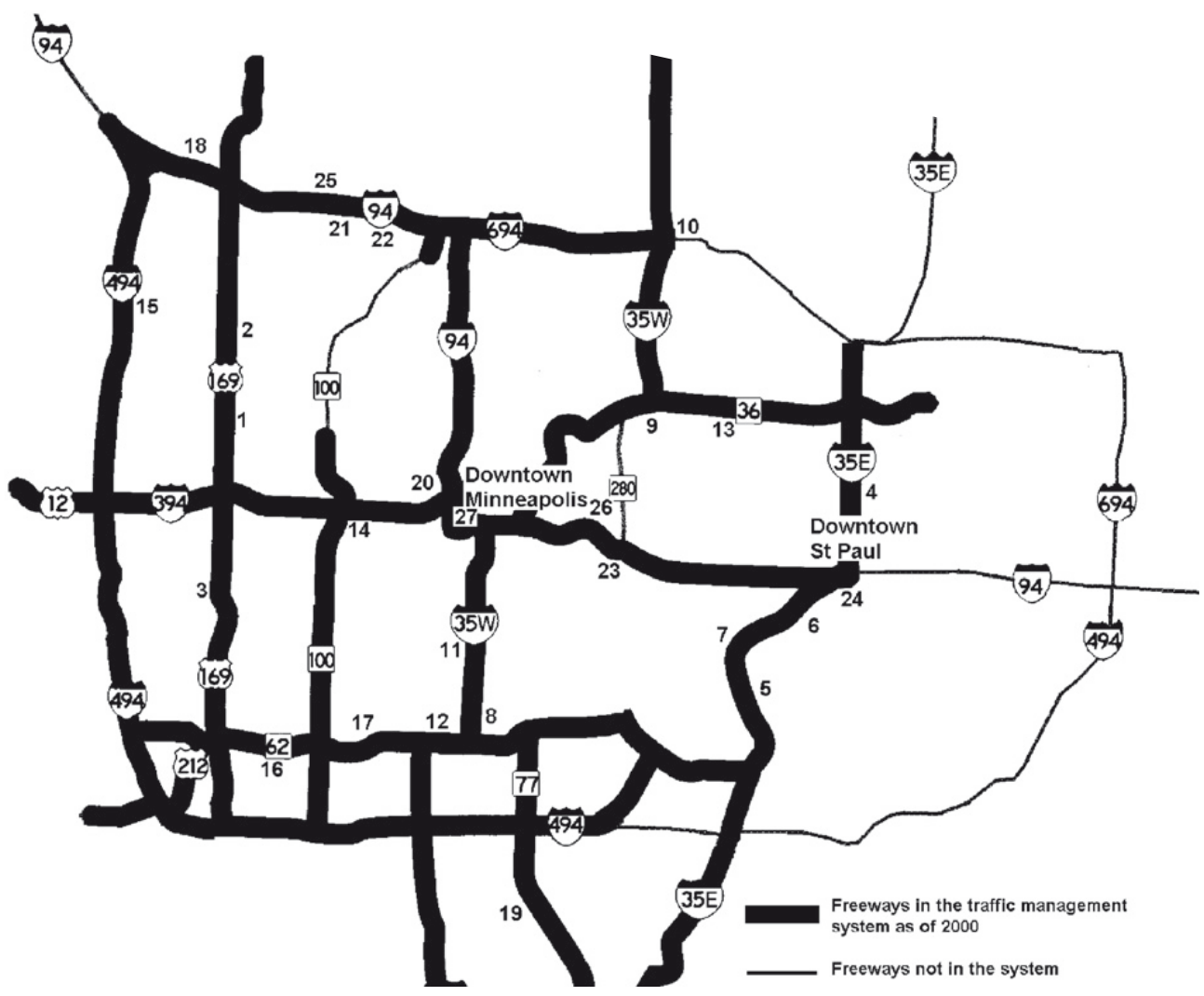

\begin{tabular}{llll}
\hline Id & Freeway & Location & Type \\
\hline 1 & TH169 NB & TH55 & Weaving section \\
2 & TH169 NB & Medicine Lake Rd & Entrance ramp \\
3 & TH169 SB & Cedar Lake Rd & Entrance ramp \\
4 & I-35E NB & Arlington Ave & Entrance ramp \\
5 & I-35E NB & Shepard Rd & Bridge, Entrance ramp \\
6 & I-35E NB & Ramsey-Grand & Entrance ramp \\
7 & I-35E SB & St Clair Ave & Entrance ramp \\
8 & I-35W NB & 50th St & Entrance ramp \\
9 & I-35W NB & TH36 & Weaving section \\
10 & I-35W NB & I-694 & Weaving section \\
11 & I-35W SB & 46th St & Entrance ramp \\
12 & I-35W SB & TH62 & Weaving section, Horizontal curve \\
13 & TH36 EB & Lexington Ave & Entrance ramp \\
14 & I-394 EB & TH100 & Entrance ramp \\
15 & I-494 NB & 49th Ave & Vertical curve, Entrance ramp \\
16 & TH62 EB & Tracy Ave & Horizontal curve, Entrance ramp \\
17 & TH62 WB & Valley View Rd & Horizontal curve, Entrance ramp \\
18 & I-694 WB & CR61 & Entrance ramp \\
19 & TH77 SB & Old Shakopee Rd & Bridge, Entrance ramp \\
20 & I-94 EB & Hennepin Ave & Tunnel, 3-d curve, Entrance ramp, \\
21 & I-94 EB & CR81 & Horizontal curve, Entrance ramp, \\
22 & I-94 EB & CR152 & Entrance ramp \\
23 & I-94 EB & TH280 & 3-d curve, Entrance ramp \\
24 & I-94 EB & I-35E & Weaving section \\
25 & I-94 WB & CR81 & Lane drop 3 to 2 \\
26 & I-94 WB & Riverside Ave & 3-d curve, Entrance ramp \\
27 & I-94 WB & Hennepin Ave & Tunnel, 3-d curve, Entrance ramp \\
\hline & & &
\end{tabular}

Fig. 1. Location of the studied bottlenecks.

This allows us to gather flow and occupancy data no further than 0.4-km (a quarter mile) upstream and downstream of studied bottlenecks. 


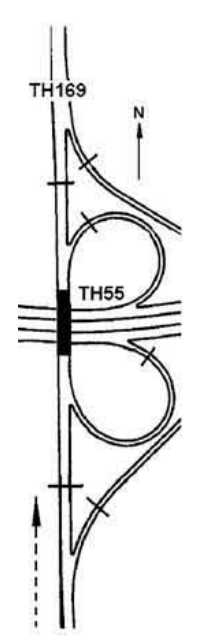

1

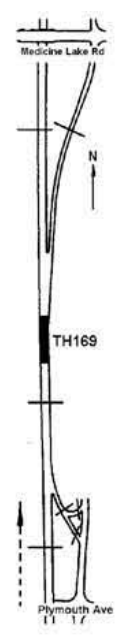

2

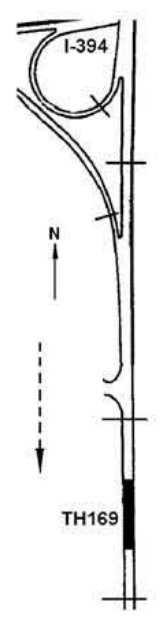

3

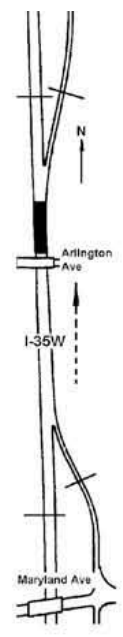

4

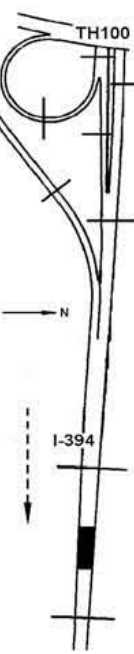

14

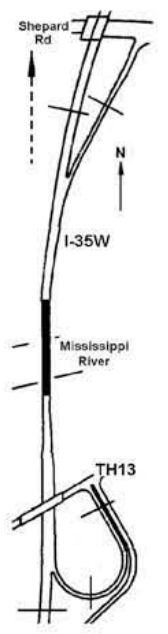

5
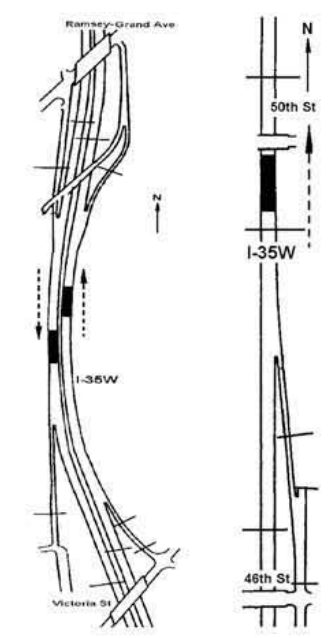

6 and $7 \quad 8$
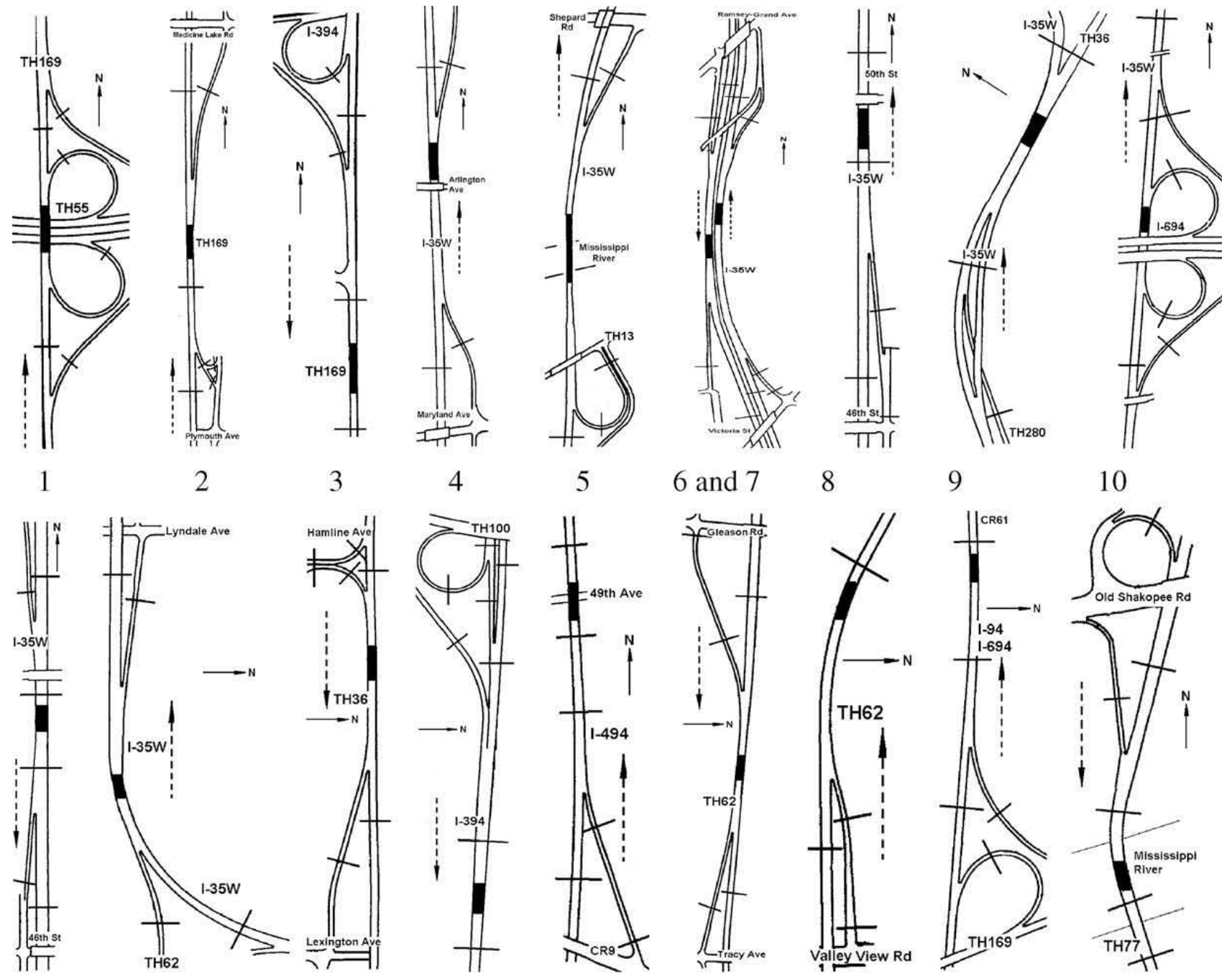

12

13

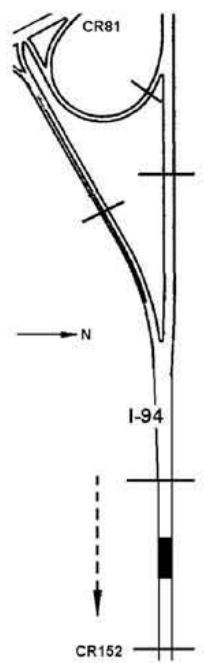

22

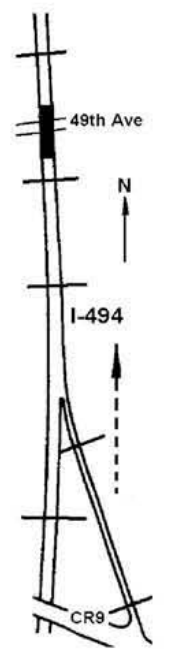

15

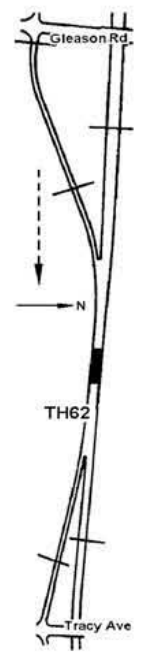

16

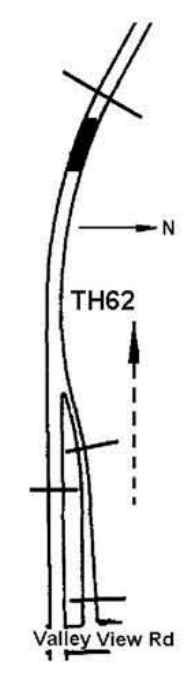

17
9

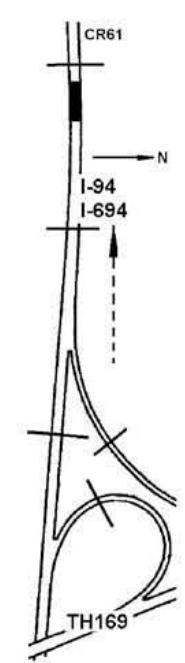

10

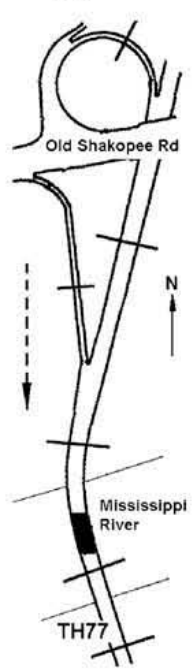

19

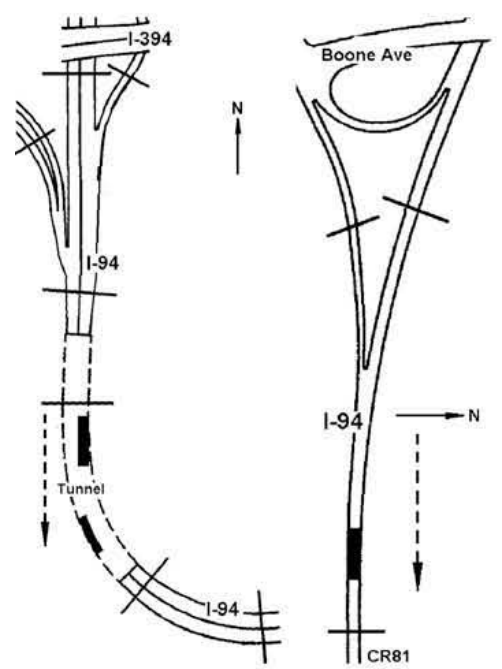

20 and 27 21

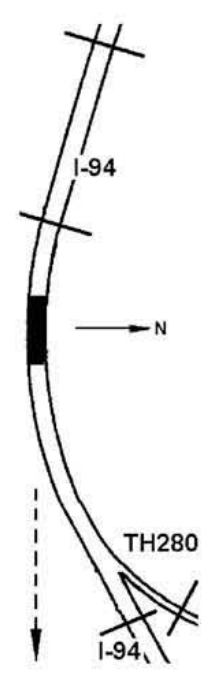

23

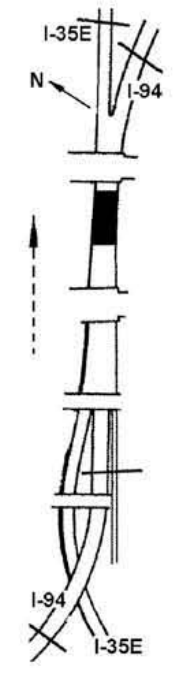

24

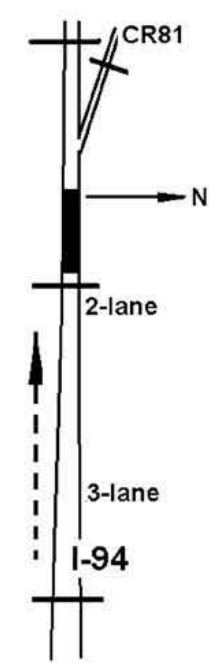

25

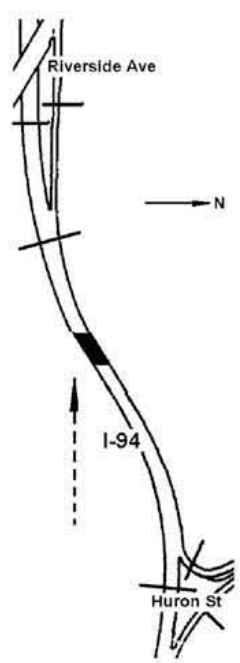

26

Bottleneck

- Detector Station

Direction of travel

Fig. 2. Studied freeway bottlenecks. Source: Minnesota Department of Transportation (2000). 


\section{Development of hypotheses}

Breakdown refers to the transition of traffic from the uncongested state where small disturbances do not affect upstream traffic to the congested state at bottlenecks. After breakdown, a queue forms upstream of the bottleneck while the flow downstream remains uncongested. This state of a bottleneck is said to be active (Daganzo, 1997). On a time-series plot, breakdown is often associated with a sharp speed drop and sometimes a flow drop during a period of high demand. Two typical profiles of flow collected just downstream of a bottleneck without metering are plotted in Fig. 3a (with breakdown) and in Fig. 3b (without breakdown). In Fig. 3a, the demand at the bottleneck increases at the beginning of the peak period. At time $t_{s}$, the flow equals the long-run average queue discharge flow of the bottleneck, $q_{d, \text { off. }}$ The subscript denotes metering status (off or on). Then after a period of high flows $\left(T_{\text {off }}\right.$ ), at time $t_{b}$ a breakdown activates the bottleneck. We refer to $T_{\text {off }}$ as the pre-queue transition period in the remainder of the paper. The average flow rate during $T_{\text {off }}$ is $q_{a, o f f}$. After $t_{b}$ the bottleneck operates at queue discharge flow rates (QDF) until it recovers at $t_{e}$ or is deactivated by downstream congestion (this later deactivation scenario is not shown in Fig. 3). After $t_{e}$, the location may or may not experience another breakdown. Fig. $3 \mathrm{~b}$ shows another possible flow pattern at a bottleneck without metering. Again, as the demand increases, $t_{s}$ will be observed. But the location does not experience a breakdown in this case possibly due to short duration of high demand or simply by chance. The duration of $T_{\text {off }}$ and $q_{a, o f f}$ can be similarly identified in this case, but not the QDFs. It should be noted that the prequeue transition flow, $q_{a}$, computed under our definition is lower than that in previous papers (e.g. Cassidy and Bertini, 1999; Hall and Agyemang-Duah, 1991; Banks, 1991a,b), because our approach takes into account both the rising and falling flows during the pre-queue transition period, not just the highest flow rate. Therefore, the readers should be aware that our method underestimates the flow drop following bottleneck breakdowns compared to previous findings.

If ramp meters control the freeway where the bottleneck is located, one can also find the two typical flow profiles, one with and the other without breakdown (Fig. $3 c$ and d). Likewise, the average QDF $\left(q_{d, o n}\right)$, the duration of the pre-queue transition period $\left(T_{o n}\right)$, and the average flow rate during the transition period $\left(q_{a, o n}\right)$ can be obtained. In those schematic graphs, one may notice a long pre-queue transition period $T$, constant QDF, $q_{a}$ significantly higher than $q_{d}$, and $q_{d, \text { on }}$ significantly higher than $q_{d, o f f}$. All these are for the sake of illustration and their real characteristics and relationships are subject to subsequent statistical tests.

\subsection{Hypothesis 1: $T_{\text {on }}$ and $T_{\text {off }}$ are not negligible}

If this hypothesis is rejected by data, it implies whenever the demand at a bottleneck exceeds its $q_{d}$, breakdown occurs and the bottleneck is activated. Therefore, the rejection of the hypothesis will also reject the two-capacity hypothesis that the flow at an active bottleneck drops after the queue formation upstream since the pre-queue flows have almost never been higher than $q_{d}$ for a meaningful amount of time. Previous studies (Hall and Agyemang-Duah, 1991; Persaud et al., 1998; Cassidy and Bertini, 1999) show the duration of the transition period without ramp metering ranges from 3 to 32 min, though different definitions of the transition period $T$ are used. Also worth noting in these studies is that only transition periods followed by breakdowns are considered (Fig. 3a) while transition periods not followed by breakdowns (Fig. 3b) are ignored, which may underestimate $T$.
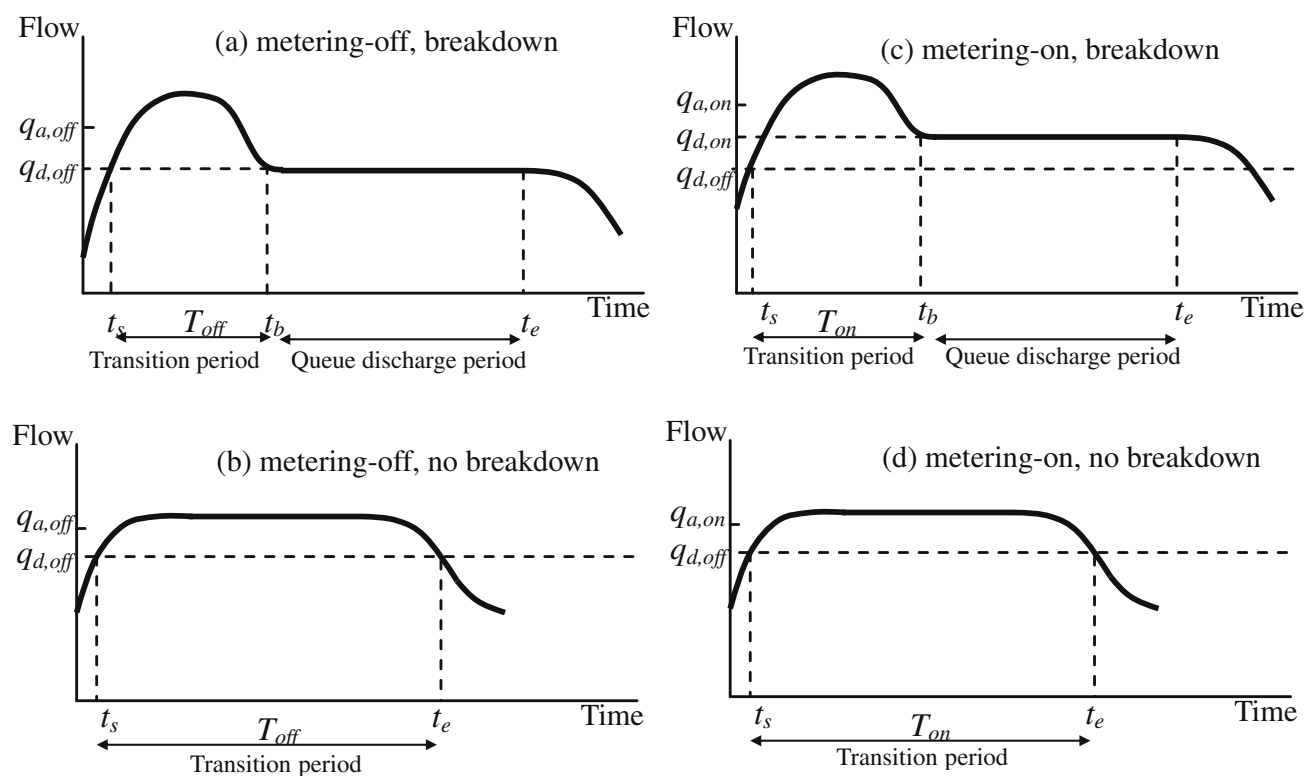

Note: $q_{a}$ is the average flow rate during $T$.

Fig. 3. Possible flow profiles at an active bottleneck with and without ramp metering. 


\subsection{Hypothesis 2: $q_{a, o f f}>q_{d, o f f}$ and $q_{a, o n}>q_{d, o n}$}

This hypothesis asserts that the average flow rate during the pre-queue transition period is significantly higher than the average queue discharge flow. Past studies have shown mixed results on this test, a summary of which is available in Cassidy and Bertini (1999).

\subsection{Hypothesis 3: $T_{\text {on }}>T_{\text {off }}$}

This hypothesis states that ramp metering can prolong the pre-queue transition period, and delay or even eliminate breakdowns. It has been conjectured in several previous studies (e.g. Banks, 1991a,b), and often cited as a rationale for ramp metering. It has not been statistically tested against empirical data with and without an operational ramp control strategy.

\subsection{Hypothesis 4: $q_{a, o n}>q_{a, o f f}$}

This hypothesis states that the average flow rate during the pre-queue transition period is higher with metering than without. A short reflection would suggest that $q_{a, o n}$ is most likely to be equal to $q_{a, \text { off }}$, because when the freeway operates at the free-flowing condition during $T_{\text {off }}$ and $T_{o n}$, the average flow rates should only depend on demand patterns. The hypothesis, if supported by the data, implies the breakdown probability at the same demand level depends on ramp control status (lower with metering).

\subsection{Hypothesis 5: $q_{d, \text { on }}>q_{d, \text { off }}$}

Several previous studies, using data either with or without metering, suggest that queue discharge flows at an active bottleneck are relatively constant over time (e.g. Cassidy and Bertini, 1999). If a breakdown occurs with metering, $q_{d, o n}$ may be improved resulting from smoothed merging maneuvers and thus higher than $q_{d, \text { off. }}$.

\subsection{Hypothesis 6: ramp metering can increase the capacity of freeway bottlenecks}

If hypotheses 3-5 are not rejected, hypothesis 6 is corroborated under any existing definition of capacity. It will be rejected if all the above three are rejected. Otherwise, further tests are required. A specific definition of capacity is not given in this paper. Of course, a standard definition of capacity, as recommended by the Highway Capacity Manual may be adopted here, based on which hypothesis 6 can be tested against empirical data even without the previous five tests. However, we feel that this would unnecessarily reduce the value of the results. The impact of ramp metering on properties of pre-queue transition flows, duration of pre-queue transition periods, and queue discharge flows is essential for us to understand how ramp metering affects traffic conditions at freeway bottlenecks.

A $t$-test on the paired differences of two metering scenarios (one pair for each bottleneck) is performed to evaluate hypotheses 2-5. Hypothesis 1 will only be evaluated empirically since a statistical test on $T>0$ will never be rejected and cannot provide any additional information. The only two assumptions of the paired-difference $t$-test are that the sample differences are randomly selected and the distribution of the population of paired differences is normal. The first assumption should be satisfied because the 27 bottlenecks used in the study can be viewed as a random sample of all bottlenecks in the Twin Cities. The normality assumption for each hypothesis test is double-checked using the Shapiro-Wilk normality test (Shapiro and Wilk, 1965) and the normal probability plot. The test results show that all five normality assumptions (one for each $t$-test) cannot be rejected ( $p$-values range from 0.16 to 0.94 ).

\section{Methodology}

An active bottleneck is characterized by a queue upstream and uncongested flow conditions downstream. The foremost task of this section is to develop a reliable and effective method that can identify active bottlenecks by checking traffic conditions upstream and downstream of every freeway segment with valid data in the Twin Cities metro area during the two seven-week study periods. Section 4.1 summarizes several existing data diagnostic tools. The following Section 4.2 explains why an improved occupancy-based method is selected. Section 4.3 describes the occupancy-based method with two thresholds in detail, followed by a discussion on the calibration of the two occupancy thresholds (Section 4.4 ). Section 4.5 compares the proposed method with an established diagnostic tool - transformed cumulative count curves (Cassidy and Windover, 1995).

\subsection{Summary of existing methods for identifying active bottlenecks}

Daganzo (1997) summarizes three methods for the identification of active freeway bottlenecks. If speed measurements are available, an increase in average point speed along the traffic flow direction indicates a possible bottleneck (speed method). Similarly a decrease in occupancy or density along the direction of travel is also a practical indicator of active bottlenecks 
(occupancy method). The third method is due to Cassidy and Windover (1995) who check that waves on both sides of the bottleneck propagate away from it using transformed cumulative flow and occupancy curves (wave method). Other traffic characteristics have also been used in past studies to identify freeway breakdown, e.g. occupancy-volume ratio (Hall and Agyemang-Duah, 1991) and pure visual inspection of time-series plots (Persaud et al., 1998).

For both speed and occupancy methods, Daganzo (1997) warns that without information about the optimal speed or occupancy thresholds, a drop of those measures may also be detected due to a fast- or slow-moving platoons within a long queue. If not applied appropriately, these two methods could misdiagnose segments within long freeway queues as active bottlenecks, because each interchange engulfed in such a queue may exhibit a higher on-ramp flow than off-ramp flow (Cassidy and Mauch, 2001; Windover and Cassidy, 2001). The advantage of speed and occupancy methods is that the whole checking process can be automated and the algorithm does not involve further subjective judgment after the threshold values are determined. The wave method keeps track of vehicle accumulation and is the most reliable of all existing data diagnostic tools. In addition, the wave method is able to reveal some traffic features that cannot be displayed in other diagnostic methods. However, it requires for each breakdown occurrence visual identification of the start and end time of the pre-queue transition and queue discharge periods. The number of freeway sections under consideration in this study is on the order of several hundred, which is required to systematically identify active bottlenecks in a metropolitan area. For a study period of 14 weeks, there are more than a thousand breakdown occurrences that need to be analyzed. Therefore, the superior reliability of the wave method and the efficiency of the speed or occupancy method are both attractive. There are several choices: (1) Use the wave method, but examine only several bottlenecks instead of 27 and significantly shorten the study period to a few days instead of 14 weeks; (2) develop an expert system that first induces the visual inspection rules human researchers use, and then apply the rules to automatically execute the wave method; (3) improve the reliability of the speed or occupancy method while keeping their efficiency.

\subsection{Selection of the data diagnostic tool}

The impacts of ramp metering on different types of bottlenecks could be quite different. It is thus crucial for this study to include various types of bottlenecks with different geometry, traffic conditions and number of lanes. A previous study (Levinson and Sheikh, 2002) concludes that although after the meters were shut-off in the Twin Cities freeway traffic clearly but slowly moves to a new equilibrium. Even at the end of the shut-off experiment, traffic fluctuation compared to historical data was still slightly higher. From this point of view, a longer study period would be desirable. Option 1 is eliminated for those reasons. The idea in option 2 is interesting and probably deserves a separate research project in its own right. Preliminary analysis of such an expert system suggests that data noise issues may pose a hurdle. Therefore, the final decision is to improve the reliability of the occupancy method and validate the improved method by comparing it against the wave method for at least one bottleneck occurrence at each studied bottleneck.

The single loop detectors installed on Twin Cities freeways only provide flow and occupancy readings. To obtain speed estimates, the average vehicle length, which is a dynamic function of traffic composition, is needed. Such a step may introduce biases. Therefore, the occupancy method is selected over the speed method, though both methods should be about equally reliable. We understand that the validity of the traditional occupancy method based on a single threshold is questionable when they are used to detect active bottlenecks. A major improvement we propose is the adoption of two occupancy thresholds, thus creating a buffer zone to deal with the spatial (across bottlenecks due to sensitivity settings) and temporal variations (at the same bottleneck due to changing traffic composition) of the optimal occupancy threshold values.

\subsection{A method for identifying active bottlenecks based on two occupancy thresholds}

This section illustrates how the proposed occupancy-based method identifies active bottlenecks, and derives the average queue discharge flow $\left(q_{d}\right)$, duration of pre-queue transition periods $(T)$, and average pre-queue transition flow $\left(q_{a}\right)$ for testing hypotheses developed in Section 3.

The general guideline in the development of this occupancy-based bottleneck identification algorithm is a conservative one - minimize the possibility that an inactive bottleneck is diagnosed as an active bottleneck. A freeway mainline detection station consists of several loop detectors with each corresponding to one lane. If the minimum occupancy of all lanes is above 25 , which approximately corresponds to the density of $39 \mathrm{veh} / \mathrm{lane} / \mathrm{km}$ with $5 \%$ trucks, the detection station is considered as congested within a queue. If the maximum occupancy of all lanes is below 20 (approximately 31 veh/lane/km; Section 4.4 explains why 25 and 20 are chosen as the thresholds), the detection station is considered as uncongested. Time intervals belonging to neither of the above two states are intermediate periods. During the intermediate periods which are usually very short, breakdowns occur, and queues build up from one lane to all lanes, or from the bottleneck to the closest upstream station. Data analysis suggests that the duration of an intermediate period is almost never longer than 10 min. Therefore, all intermediate periods are simply excluded from the following computation of traffic parameters. This will slightly reduce the length of computed queue discharge periods and possibly pre-queue transition periods, but should not bias the hypothesis testing results in any significant way (see period 3 in Fig. 4 for an example; note only the time-series plot on the top of Fig. 4 is used for illustration in this section). 
Each freeway station in each 32 time interval must be under one of the following three conditions according to the occupancy readings across all lanes: congested, uncongested, or intermediate. Then each pair of adjacent freeway mainline stations can be examined.

If the upstream station is congested while the downstream one is uncongested for more than five consecutive minutes, the freeway mainline section between the two stations is considered as an active bottleneck. The only exception is that the queue at the upstream station may be caused by insufficient off-ramp capacity if there is an off-ramp between the two sta-

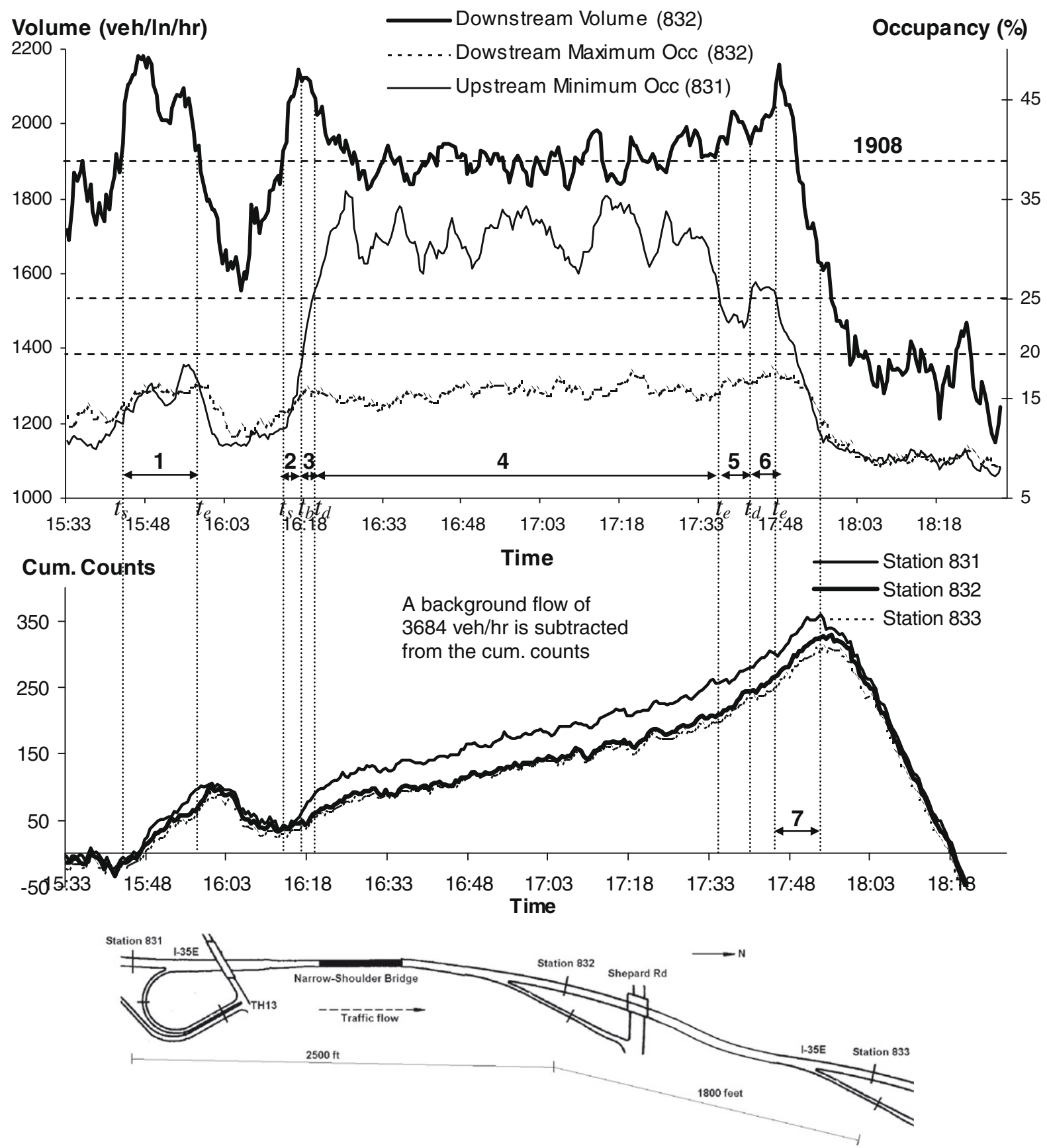

$1908 \mathrm{veh} / \mathrm{ln} / \mathrm{hr}$ : long-run average queue discharge flow rate calculated from the 7-week study period;

1: The first observed pre-queue transition period ( 15 minutes, not followed by a breakdown);

2: The second pre-queue transition period (4 minutes, followed by a breakdown);

3: Intermediate period excluded from the analysis ( 3 minutes);

4: The first queue discharge period (1 hour 17 minutes);

5: A period lost due to conservative occupancy thresholds (5 minutes);

6: Another queue discharge period (5 minutes);

7: The end of the dissipating queue is between the bottleneck and station 831 during this period.

Fig. 4. Comparison of two data diagnostic methods (Bottleneck 5, November 21, 2000). 
tions (a diverge bottleneck). Therefore, traffic conditions at the off-ramp must also be considered. If the off-ramp station is congested, the freeway mainline section is not an active bottleneck because what is critical in this case is the capacity of the off-ramp, not the mainline section. Diverge bottlenecks are excluded from the analysis because it is unlikely that meters would have any impacts on the capacity of off-ramps (which actually depends on traffic and control on local streets). It should be noted that depending on the placement of the off-ramp detectors, a diverge bottleneck may be active even when the off-ramp detection station is not congested. This is caused by mainline exiting flow rates exceeding off-ramp capacity. Therefore, caution should be taken if the above method for identifying active freeway bottlenecks is applied to freeway locations with off-ramps carrying high mainline exiting flows.

Once an active freeway bottleneck is identified, QDFs will be collected immediately downstream of the bottleneck until it is deactivated (see period 4 in Fig. 4). If both the upstream and the downstream stations return to the uncongested state for more than $5 \mathrm{~min}$, the beginning of that 5-min period is considered as the end of the preceding queue discharge period. There may be multiple queue discharge periods during a peak period. The average QDF of each afternoon peak period with and without ramp metering can be calculated $\left(q_{d, \text { off }}\right.$ and $\left.q_{d, o n}\right)$. When flows in queue discharge periods across all studies days are averaged, the long-run average QDF at each bottleneck is obtained. It should be mentioned that some studied freeway sections never experienced breakdown when meters were on in 1999. The variable $q_{d, \text { on }}$ is hence not available at these locations (14 out of 27 bottlenecks). The total number of queue discharging periods with and without ramp metering respectively are also recorded because they disclose whether ramp metering reduces breakdown occurrences.

If both the upstream and the downstream stations of a bottleneck are uncongested and the flow measured at the downstream station is higher than the long-run average QDF during a 32 interval, that interval is a part of the pre-queue transition period $T$ because the demand during that interval is high enough to potentially cause a breakdown (see period 2 in Fig. 4 ). There may be multiple pre-queue transition periods during a peak period, and a transition period may not be followed by a queue discharge period (see an example of non-breakdown transition period in Fig. 4 - period 1). Since previous studies do not consider non-breakdown transition periods and only report the average duration of breakdown transition periods, these two types of pre-queue transition periods are distinguished in the analysis to facilitate comparison to earlier work. If a prequeue transition period ends and a subsequent queue discharge period starts within 10 min (a 10-min threshold is adopted to account for the short intermediate period - such as period 3 in Fig. 4), this pre-queue transition period is associated with the breakdown. Otherwise, it is considered as a non-breakdown transition period. The total length of all transition periods ( $T$ ) throughout an afternoon peak period is computed and used in the hypothesis testing. The flow rates in the transition periods are collected by the same set of detectors that collect QDFs (i.e. the detection station immediately downstream of the bottleneck). The average flow during the transition period is $q_{a}$. The computation of $T$ and $q_{a}$ uses $q_{d, \text { off }}$ for both metering-on and -off periods, so the results with and without metering are comparable.

If the downstream station of a freeway section is congested, the traffic condition at the current section is determined by active bottlenecks further downstream. These observations are excluded from the analysis of the current freeway section. An example of such situations is included in the following section.

One conceivable drawback of the traditional occupancy method is that when a freeway section is within a long queue, a decrease in occupancy along the direction of travel may still be observed in that section because traffic entering from the onramps consumes mainline capacity (Cassidy and Mauch, 2001). Two data selection criteria are adopted in the improved method to address this issue - only bottlenecks with more than eight breakdown observations are included in the analysis, and only queue discharge periods longer than $5 \mathrm{~min}$ are considered as real queue discharge periods. The occupancies measured at a short distance upstream of a bottleneck can be relatively low during some time intervals. But the duration of the low occupancy readings ( $<20 \%$ used in this analysis) within a queue is rarely longer than 5 min across all lanes. With these two additional requirements in the occupancy method, the likelihood that a segment within a queue is misdiagnosed as active bottlenecks should be very small, if not zero.

The occupancy method uses the maximum occupancy of all lanes at the downstream station (with respect to the active bottleneck) and the minimum occupancy of all lanes at the upstream station to determine if a section is congested or not. Although this technique effectively reduces the chance of misdiagnoses, it could make the method more restrictive (requiring worse conditions) as the number of lanes increases because typically one or more lanes frequently exhibits a lower occupancy than the others during congestion. As a result of this fact, it is possible that at a mildly congested bottleneck breakdown is not detected by the occupancy method until much later and artificially extending the pre-queue discharge per$\operatorname{iod} T$. After categorizing results by number of lanes at the bottlenecks (see Note a in Table 1 in Section 5), there is no evidence that this type of misdiagnosis occurred in our analysis - the percentage increase of the pre-queue transition period is not higher at bottlenecks with more lanes. But the described situation might occur had an upper-bound occupancy threshold higher than the $25 \%$ used in this analysis been selected.

\subsection{Calibration of the two occupancy threshold values}

We sampled more than thirty breakdowns at several well-known bottlenecks on Trunk Highway 169 and I-94 in order to decide which threshold values to use in the occupancy method. Time-series occupancy and flow data, as well as cumulative count curves, were plotted for all breakdown observations in the sample in a fashion similar to Fig. 4, where the effectiveness of alternative occupancy thresholds can be tested. Visual inspection was used to identify the "optimal" threshold values on the plots. The starting point of the search for the threshold values in the experiment is $18 \%$, which is used by the Minnesota 
Table 1

Some traffic characteristics at all studied active bottlenecks with ramp metering on and off.

\begin{tabular}{|c|c|c|c|c|c|c|c|c|c|c|c|c|c|c|c|c|c|c|}
\hline \multirow[t]{2}{*}{ Id } & \multirow[t]{2}{*}{ \# Lanes ${ }^{\mathrm{a}}$} & \multicolumn{2}{|c|}{ \# Days } & \multicolumn{2}{|c|}{$\begin{array}{l}\text { Breakdown } \\
\text { per day }\end{array}$} & \multicolumn{3}{|c|}{$q_{d}(\operatorname{veh} / \mathrm{h})$} & \multicolumn{7}{|c|}{$\begin{array}{l}T(\text { hh:mm/day })^{\mathrm{b}} T_{b}(\mathrm{~mm} / \text { breakdown period }) T_{n b}(\mathrm{~mm} / \\
\text { non-breakdown period) }\end{array}$} & \multicolumn{3}{|c|}{$q_{a}($ veh/h $)$} \\
\hline & & Off & On & Off & On & Off & On & $\Delta(\%)$ & Off $T_{b}$ & Off $T_{n b}$ & On $T_{b}$ & On $T_{n b}$ & Off $T$ & On $T$ & $\Delta(\%)$ & Off & On & $\Delta(\%)$ \\
\hline 1 & 2 & 24 & 29 & 3.1 & 1.6 & 4152 & 4349 & 5 & 9 & 7 & 37 & 45 & 0:13 & $1: 21$ & 523 & 4237 & 4419 & 4 \\
\hline 2 & 2 & 26 & 29 & 2.1 & 0.4 & 3991 & 3997 & 0 & 18 & 13 & 86 & 77 & $1: 08$ & $2: 37$ & 131 & 4223 & 4328 & 2 \\
\hline 3 & 2 & 27 & 24 & 0.3 & 0 & 4107 & NA & NA & 10 & 8 & NA & 19 & $0: 27$ & $0: 35$ & 30 & 4256 & 4298 & 1 \\
\hline 4 & 3 & 28 & 29 & 0.3 & 0.2 & 5885 & 6115 & 4 & 27 & 30 & 8 & 92 & $1: 54$ & $2: 33$ & 34 & 6396 & 6490 & 1 \\
\hline 5 & 2 & 27 & 29 & 0.8 & 0 & 3816 & NA & NA & 30 & 10 & NA & 18 & $1: 08$ & $1: 03$ & -7 & 4052 & 4078 & 1 \\
\hline 6 & 2 & 26 & 29 & 1.2 & 0 & 3900 & NA & NA & 5 & 6 & NA & 16 & $0: 22$ & $1: 11$ & 223 & 4016 & 4042 & 1 \\
\hline 7 & 2 & 27 & 29 & 0.4 & 0 & 4019 & NA & NA & 4 & 11 & NA & 24 & $0: 44$ & $1: 07$ & 52 & 4179 & 4212 & 1 \\
\hline 8 & 3 & 28 & 28 & 0.9 & 0.5 & 5694 & 5679 & 0 & 18 & 22 & 32 & 56 & $2: 59$ & $3: 18$ & 11 & 6156 & 6165 & 0 \\
\hline 9 & 4 & 27 & 28 & 0.4 & 0 & 8336 & NA & NA & 14 & 8 & NA & 73 & $0: 36$ & $2: 03$ & 242 & 8837 & 9291 & 5 \\
\hline 10 & 3 & 27 & 29 & 2.1 & 0 & 6728 & NA & NA & 9 & 10 & NA & 82 & 0:04 & $1: 27$ & 2075 & 6885 & 7329 & 6 \\
\hline 11 & 3 & 26 & 28 & 0.7 & 0 & 6650 & NA & NA & 8 & 8 & NA & 18 & $1: 13$ & $1: 58$ & 62 & 6851 & 6892 & 1 \\
\hline 12 & 3 & 27 & 28 & 0.7 & 0.8 & 5243 & 5079 & -3 & 13 & 13 & 14 & 20 & $3: 05$ & $1: 34$ & -49 & 5490 & 5389 & -2 \\
\hline 13 & 2 & 27 & 28 & 0.5 & 0 & 4206 & NA & NA & 15 & 13 & NA & 16 & $0: 40$ & $0: 49$ & 23 & 4430 & 4304 & -3 \\
\hline 14 & 3 & 27 & 29 & 2.6 & 1.8 & 5196 & 5393 & 4 & 14 & 10 & 38 & 30 & 1:04 & $1: 36$ & 50 & 5450 & 5396 & -1 \\
\hline 15 & 2 & 26 & 29 & 1.3 & 0 & 4165 & NA & NA & 14 & 8 & NA & 22 & $0: 14$ & $1: 24$ & 500 & 4264 & 4313 & 1 \\
\hline 16 & 2 & 24 & 29 & 1.2 & 0 & 4004 & NA & NA & 15 & 12 & NA & 23 & $0: 38$ & $1: 19$ & 108 & 4258 & 4240 & 0 \\
\hline 17 & 2 & 26 & 29 & 0.5 & 0 & 3876 & NA & NA & 5 & 6 & NA & 16 & $1: 08$ & $1: 14$ & 9 & 4028 & 3997 & -1 \\
\hline 18 & 2 & 23 & 28 & 2.2 & 1.0 & 3867 & 3882 & 0 & 6 & 11 & 30 & 49 & $0: 53$ & $1: 54$ & 115 & 4054 & 4161 & 3 \\
\hline 19 & 3 & 27 & 29 & 0.2 & 0 & 6122 & NA & NA & 3 & 11 & NA & 45 & $0: 49$ & $1: 12$ & 47 & 6418 & 6580 & 3 \\
\hline 20 & 3 & 28 & 9 & 0.3 & 0 & 5415 & NA & NA & 50 & 18 & NA & 61 & $1: 43$ & $2: 28$ & 44 & 5664 & 5771 & 2 \\
\hline 21 & 2 & 26 & 28 & 1.7 & 0.5 & 3670 & 3599 & -2 & 10 & 9 & 46 & 29 & 1:05 & $2: 14$ & 106 & 3865 & 3846 & 0 \\
\hline 22 & 2 & 28 & 28 & 0.8 & 0.2 & 3588 & 3793 & 6 & 32 & 21 & 0 & 103 & $2: 36$ & $3: 41$ & 42 & 3914 & 4008 & 2 \\
\hline 23 & 3 & 27 & 29 & 2.3 & 1.4 & 6063 & 6250 & 3 & 14 & 9 & 79 & 61 & $0: 49$ & $2: 19$ & 184 & 6348 & 6559 & 3 \\
\hline 24 & 4 & 24 & 29 & 1.8 & 0.7 & 7335 & 8023 & 9 & 38 & 26 & 3 & 133 & $0: 58$ & $2: 45$ & 184 & 8136 & 8773 & 8 \\
\hline 25 & 2 & 28 & 28 & 1.3 & 1.8 & 3771 & 3911 & 4 & 31 & 12 & 107 & 58 & $1: 33$ & $2: 52$ & 85 & 4021 & 4149 & 3 \\
\hline 26 & 3 & 28 & 29 & 0.3 & 0.5 & 5205 & 5688 & 9 & $N A^{c}$ & 12 & 0 & 65 & $1: 56$ & $4: 08$ & 114 & 5655 & 5822 & 3 \\
\hline 27 & 3 & 28 & 9 & 1.2 & 0 & 5476 & NA & NA & 55 & 26 & NA & 88 & 1:03 & 3:08 & 198 & 5847 & 5979 & 2 \\
\hline Ave. & & 27 & 27 & 1.2 & 0.4 & 4981 & 5058 & 3 & 18 & 13 & 37 & 50 & 1:08 & $1: 59$ & 73 & 5257 & 5360 & 2 \\
\hline
\end{tabular}

a The percentage increase of $T$ is $82 \%$ for the fourteen 2 -lane bottlenecks, $54 \%$ for the eleven 3-lane bottlenecks, and $206 \%$ for the two 4 -lane bottlenecks.

b $T$ is the total duration of all pre-queue transition periods per day which is used for hypothesis testing. $T_{b}\left(T_{n b}\right)$ is the average duration of (non-) breakdown transition periods. $T_{b}$ and $T_{n b}$ are calculated for comparison with previous studies that do not include non-breakdown transition periods. See Section 4 for more detailed definition of those variables.

${ }^{c}$ No pre-queue transition period was observed that just preceded the seven breakdowns at bottleneck 26 when meters were off.

Department of Transportation to separate congested and uncongested flow. We found that $18 \%$ is a bit low; $20 \%$ and $25 \%$ were finally selected because this combination identifies pre-queue transition periods and queue discharge periods better than other combinations based on visual inspection (a combination is better if the high threshold value crosses the upstream minimum occupancy line and the low threshold value crosses downstream maximum occupancy line less often during an observable queue discharge period). More evidence of the superiority of this combination is provided in the following two examples. Thanks to the $5 \%$ buffer zone (20-25\%), the method performs well on different bottlenecks even with the same two threshold values. Adopting spatially dependent threshold values at different bottlenecks only improves the data diagnosis process marginally (shorter intermediate periods which are already very short); and still does not address temporal variations. The two threshold values were later validated using at least one breakdown observation from all 27 studied bottlenecks.

\subsection{Comparison against the wave method}

The improved occupancy method is compared with the wave method (Cassidy and Windover, 1995) at the studied bottlenecks and two representative examples are shown below to demonstrate its reliability. The first example is still at bottleneck 5 on November 21, 2000 (meters are off) as illustrated in Fig. 4.

The bottleneck is located on I-35E in Saint Paul between stations 832 and 831, and is caused by a bridge on Mississippi River with a narrow shoulder lane and the entrance ramp from TH13. The improved occupancy method (upper graph) correctly identifies the two pre-queue transition periods (periods 1 and 2), which is verified by the cumulative count curve (lower graph). It should be noted that the cumulative count curve also shows the existence of two transient queues upstream of the bottleneck but downstream of station 831 during period 1 . This feature cannot be detected on the time-series plot. But clearly, the bottleneck was not activated in this period. The real breakdown occurred at about 16:18. It is also now clear that during period 3, an intermediate period excluded from the analysis, the queue propagated upstream and reached station 831 at the beginning of period 4 . The occupancy method collects queue discharge flow rates in period 4 , which was indeed a part of the queue discharge period at bottleneck 5 on that day because the queue between station 831 and station 832 is evident 
and no queued vehicle existed between station 832 and station 833. Period 5 was also a part of the queue discharge period, but is not detected by the occupancy method due to low occupancy at the upstream station 831 . We found that the flow from the on-ramp at TH13 was relatively low just before and during period 5 so that the mainline traffic at station 831 could move faster and caused a temporary fast-moving low-occupancy platoon within the long queue upstream of the bottleneck. The occupancy method continued to collect queue discharge flows during period 6 while missing period 7. The end of the queue was between station 831 and the bottleneck during period 7 before it finally dissipated at about 17:55. Discarding periods 5

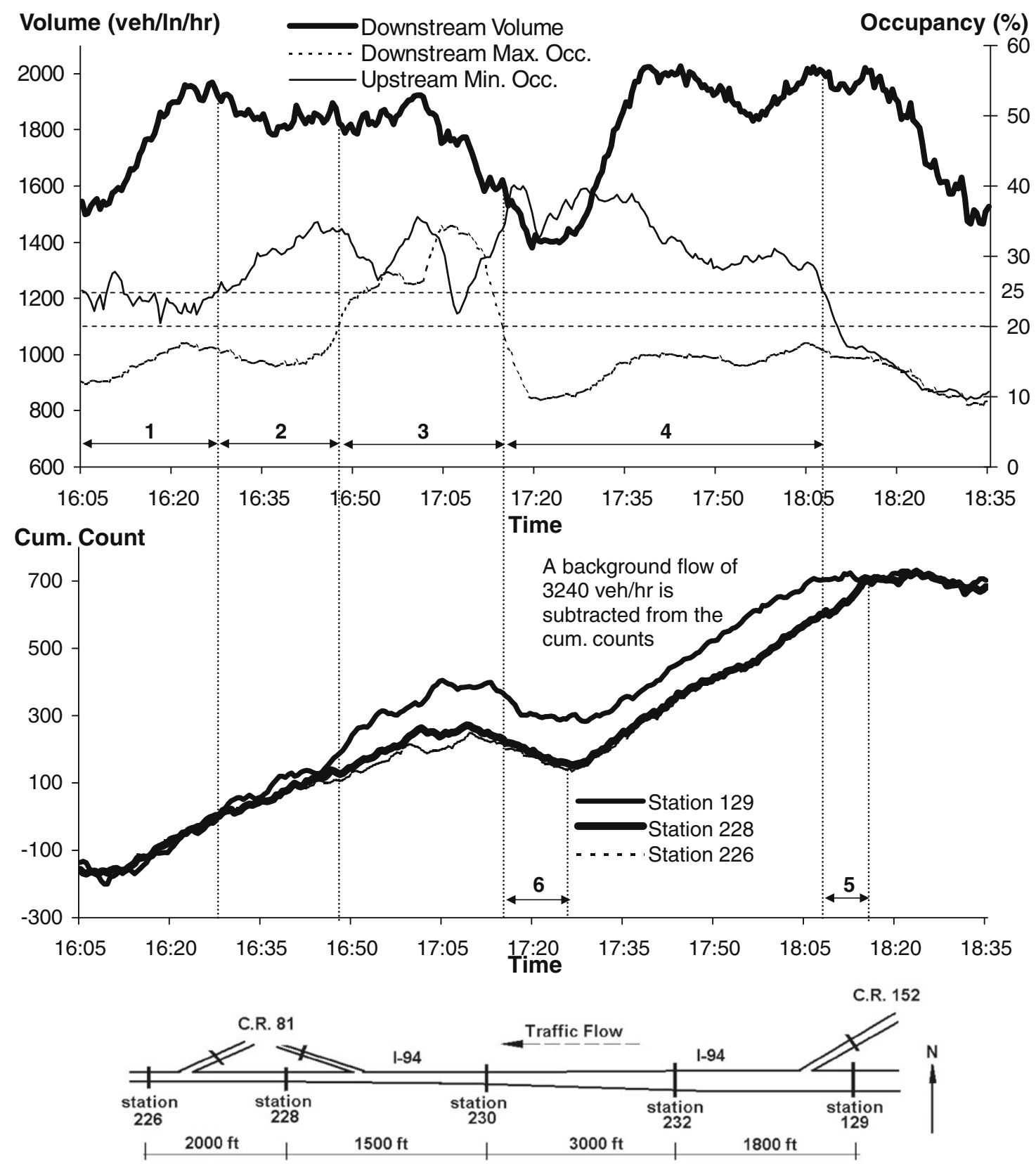

1. It is evident in this period that the occupancy method with two thresholds is better than with only one threshold;

2. Bottleneck 25 (between stations 228 and 230) is activated and display queue discharge flow;

3. Bottleneck 25 is deactivated by a more restrictive bottle neck downstream as the high occupancy values at station 228 and queuing between station 228 and station 226 both suggest;

4. Bottleneck 25 again become active after the bottleneck further downstream is relieved;

5. Bottleneck 25 is deactivated and queue dissipates between the bottleneck and station 129 during this period due to reduction of travel demand.

6. A period when the flow at bottleneck 25 recovers fro $m$ the lower queue discharger flow of the downstream bottleneck to its own queue discharge flow;

Fig. 5. Comparison of two data diagnostic methods (Bottleneck 25, November 20, 2000). 
and 7 in this particular example could cause a slight underestimation of the average queue discharge flow. It is likely that a slight overestimation might occur on some other days. But over the long run, there should not be significant systematic biases. Lastly, the low occupancy threshold, $20 \%$, performs very well in this example. An even lower threshold, such as $18 \%$, could misdiagnose station 832 as congested during periods 4 and 6 .

Let us now move to an example that is somewhat more complicated than the previous one. Bottleneck 25 in Fig. 5 is on I94 WB between stations 228 and 230 due to a lane drop from three to two lanes just upstream of station 230 . By examining the cumulative curves (lower graph) at stations 226, 228, and 129 (curves for stations 230 and 232 are not shown to improve the clarity of the graph without loss of important information), we found that on November 20, 2000 (meters were off), this bottleneck was activated at 16:29 (period 2: queue started to build up between stations 228 and 129, but not downstream of station 228), deactivated by a bottleneck further downstream at 16:45 (period 3: queue started to build up between station 226 and 228), again activated after the bottleneck further downstream was relieved around 17:20 (periods 4 and 5), and finally deactivated as demand dropped at 18:15. The reliability of the occupancy method (upper graph) is again almost equally good. It accurately identifies periods 2 and 4 when the bottleneck was active (high occupancy upstream but low occupancy downstream), and period 3 when the bottleneck was deactivated (high occupancy downstream). Using the maximum downstream occupancy across all lanes seems to be good practice to ensure that period 3 would not be misdiagnosed as a queue discharge period for bottleneck 25. The only controversy arises with respect to period 6 when the flow at bottleneck 25 recovered from the lower queue discharge flow of the further downstream bottleneck to its own higher queue discharge flow. The occupancy method considers this period as a part of the queue discharge period for bottleneck 25 . Looking at the cumulative count curve, one would agree that the impact of the bottleneck further downstream still existed during period 6. At the same time, it is also evident that the queue downstream of station 228 had already dissipated before period 6 . The behavior of that recovery wave is certainly interesting but beyond the scope of this paper. Another point worth noting in this case is the selection of the high-occupancy-threshold (25\% used in the analysis). This bottleneck location requires the highest high-occupancy-threshold, probably due to an extreme detector sensitivity setting. Let us take period 1 as an example. There was a short occupancy spike in period 1. It would not be considered as a queue discharge period by the occupancy method because it is shorter than 5 min, which avoids a misdiagnosis. Any high-occupancy-threshold lower than $25 \%$ would result in a misdiagnosis. Also conceivable is that if an occupancy method with a single threshold between $18 \%$ and $25 \%$ was used, it would misdiagnose period 1 as a queue discharge period. The value of the $5 \%$ buffer zone in the improved method with two occupancy threshold is evident in this example.

By using two thresholds, adding constraints on minimum shortest queue discharge periods, and analyzing only recurrent bottlenecks, the improved occupancy method described above as a tool for systematically identifying and analyzing active bottlenecks, satisfactorily marries efficiency and reliability for the purpose of this study.

\section{Results}

The actual number of days studied at the 27 bottlenecks varies due to weather, accidents, and detector failures. On average, 27 days were examined with and without metering respectively. This section interprets test results of the six hypotheses, as well as some other interesting findings.

Some traffic characteristics related to hypothesis testing are summarized in Table 1 . Detailed $t$-test results are presented in Table 2. Hypothesis 1 is supported. The average duration of transition periods per peak period $(T)$ is about 68 min without metering. It is also evident from the data that multiple breakdowns could occur during a peak period, and flow at a bottleneck could be higher than the long-run queue discharge flow for a long period of time without causing a subsequent breakdown (non-breakdown transition periods). After separating transition periods followed by breakdowns from those not followed by breakdowns, we found that the average duration of breakdown transition periods without metering (off $T_{b}$ ) is about $18 \mathrm{~min}$ (standard deviation $13 \mathrm{~min}$ ). This falls within the range found in past studies (3-32 min per breakdown) which did not consider non-breakdown transition periods.

Hypothesis $2\left(q_{a, o f f}>q_{d, \text { off }}\right.$, and $\left.q_{a, o n}>q_{d, \text { on }}\right)$ is confirmed. The definition of the pre-queue transition period determines that $q_{a}$ would be larger than $q_{d}$. What matters is really how much $q_{a}$ is larger than $q_{d}$. It is found from the data that on average $q_{a, \text { off }}$ is 5.5\% higher than $q_{d, o f f}$, and $q_{a, o n}$ is $5.8 \%$ higher than $q_{d, o n}$. These percentage flow drops observed under both control scenarios are statistically significant at level 0.01 (see the first two rows in Table 2). The flow drops at all studied bottlenecks after

Table 2

Hypothesis testing results.

\begin{tabular}{|c|c|c|c|c|c|c|c|}
\hline \multirow[t]{2}{*}{ Hypothesis } & \multicolumn{5}{|c|}{ One-tailed paired-difference $t$-test } & \multirow[t]{2}{*}{ Hypothesis confirmed? } & \multirow[t]{2}{*}{ \% Change } \\
\hline & Null $H_{0}$ & Alternative $H_{a}$ & $t$ & $p$ & $H_{0}$ rejected? & & \\
\hline$q_{a, o f f}>q_{d, o f f}$ & $q_{a, o f f}=q_{d, o f f}$ & $q_{a, o f f}>q_{d, o f f}$ & 9.14 & 0.00 & Yes $(d f=26)$ & Yes & +5.5 \\
\hline$q_{a, o n}>q_{d, o n}$ & $q_{a, o n}=q_{d, o n}$ & $q_{a, o n}>q_{d, o n}$ & 5.74 & 0.00 & Yes $(\mathrm{df}=12)$ & Yes & +5.8 \\
\hline$T_{o n}>T_{o f f}$ & $T_{o n}=T_{o f f}$ & $T_{o n}>T_{o f f}$ & 5.73 & 0.00 & Yes $(\mathrm{df}=26)$ & Yes & +73.4 \\
\hline$q_{a, o n}>\mathrm{q}_{\mathrm{a}, \mathrm{off}}$ & $q_{a, \text { on }}=q_{a, \text { off }}$ & $q_{a, o n}>q_{a, o f f}$ & 3.18 & 0.00 & Yes $(\mathrm{df}=26)$ & Yes & +2 \\
\hline$q_{d, o n}>q_{d, o f f}$ & $q_{d, o n}=q_{d, o f f}$ & $q_{d, o n}>q_{d, o f f}$ & 2.54 & 0.01 & Yes $(\mathrm{df}=12)$ & Yes & +3 \\
\hline
\end{tabular}




\section{Flow Drop}

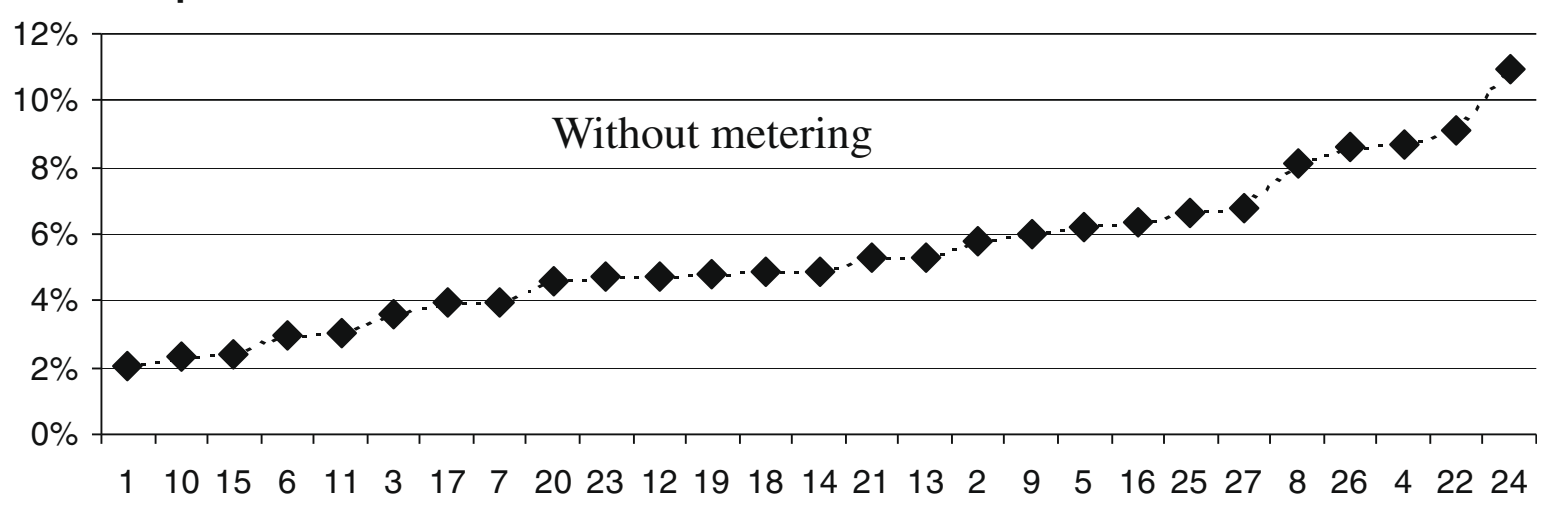

Bottleneck Id

\section{Flow Drop}

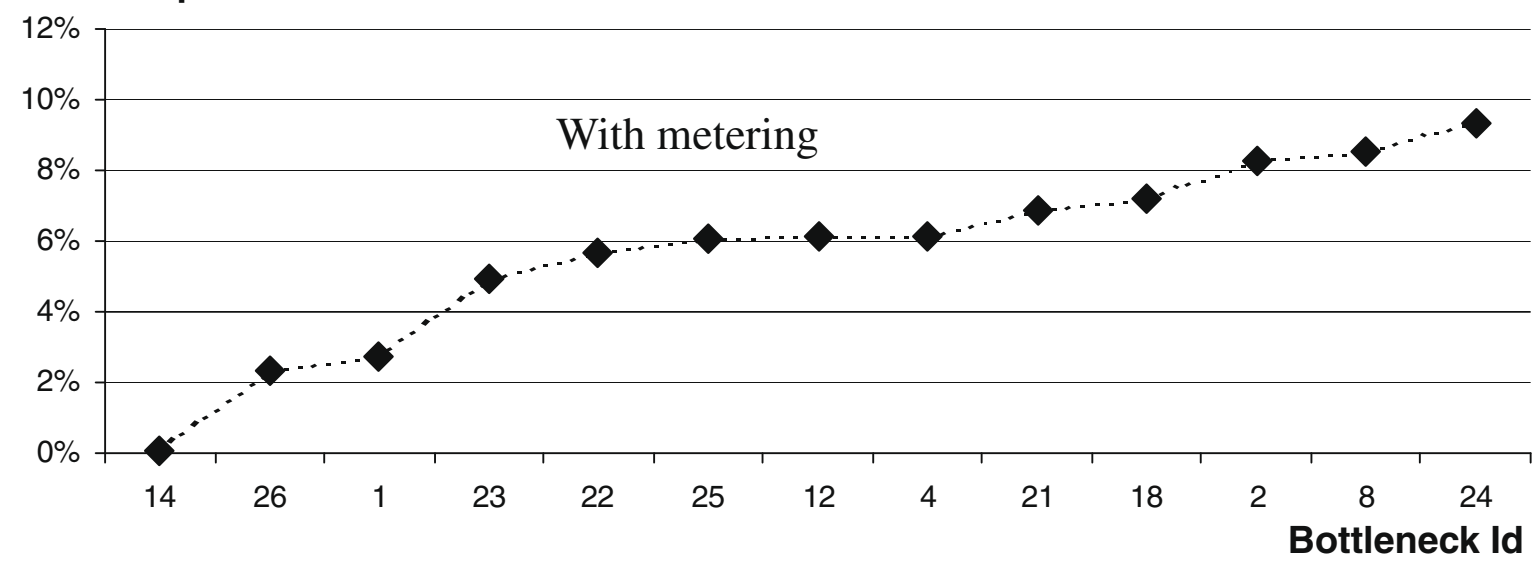

Note: Only thirteen bottlenecks still experienced breakdown with ramp metering.

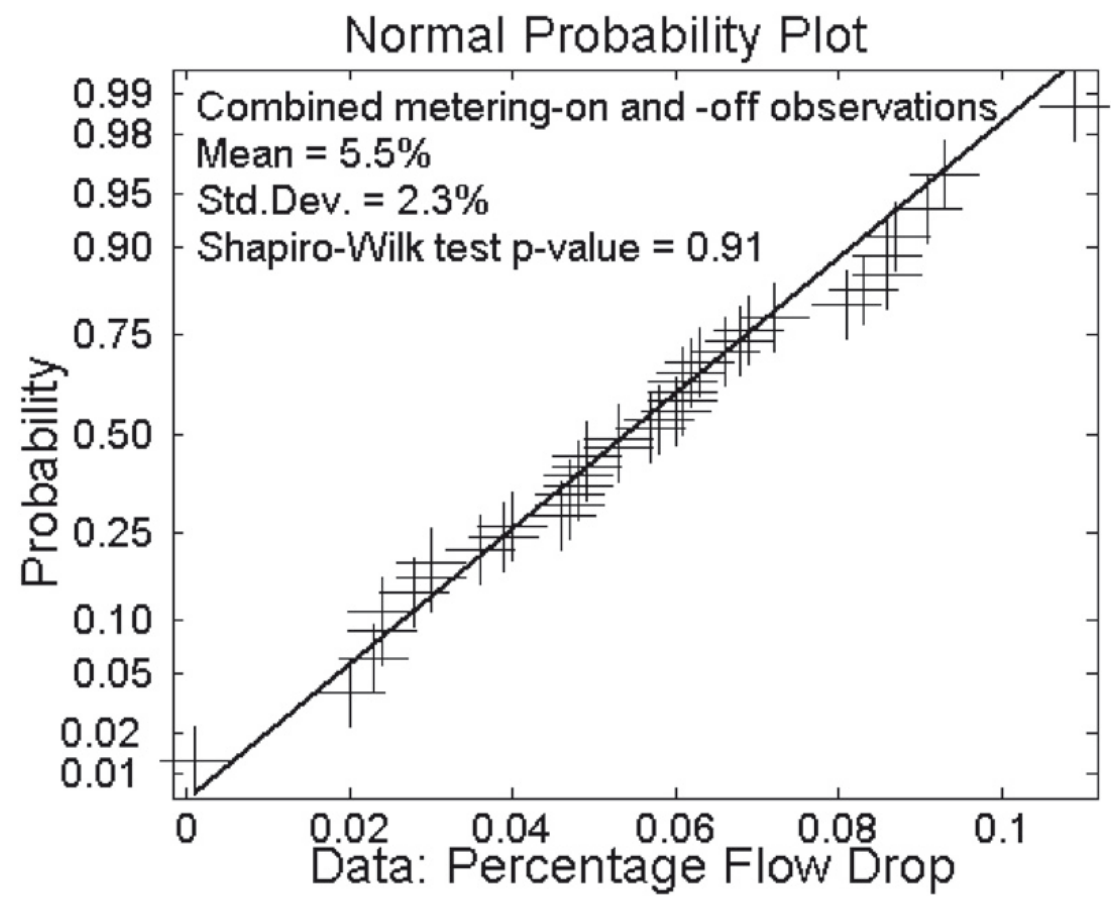

Fig. 6. Flow drop at active bottlenecks after breakdown with and without ramp metering. 
not capacity drops. Additional test results strongly suggest that the percentage flow drops at various bottlenecks are normally distributed (Normality can not be rejected at level 0.91 according to the Shapiro-Wilk test; see Fig. 6). Previous studies have drawn conflicting conclusions on this issue. Some found high percentage flow drops and considered their findings as evidence of the two-capacity hypothesis (Banks, 1991a,b; Hall and Agyemang-Duah, 1991). Others observed very small flow differences and concluded that even if the two-capacity phenomenon ever exists, it does not provide a rationale for ramp metering (Newman, 1961; Newman et al., 1969; Persaud, 1986; Persaud and Hurdle, 1991). Our results suggest that insufficient sampling from the underlying normal distribution may lead to inconsistent conclusions because no more than four bottlenecks were examined in the cited research. If this argument is true, it becomes important to understand what factors contribute to the normal variability. In that case, detailed analysis at individual bottlenecks is necessary. An alternative view is offered by Cassidy and Bertini (1999), who attribute the inconsistency to the inadequate data analysis methods used in those studies.

Whether meters are able to prolong the pre-queue transition period is examined by hypothesis 3 ( $T_{\text {on }}>T_{\text {off }}$ ). The average duration of transition periods in an afternoon peak period increases by 73\% from 68 min without metering to almost two hours with metering. The magnitude of such increase varies from location to location, but is statistically significant at level 0.01 . The average number of breakdown occurrences has also been reduced from 1.2 without metering to 0.4 per bottleneck per afternoon peak period with metering. According to these results, the ramp control strategy is able to not only extend transition periods and delay bottleneck activations, but also eliminate a significant number of breakdowns. At two bottlenecks (5, a bridge, and 12 a weaving section where I-35E southbound and TH62 westbound join), the transition periods are actually shorter with metering, which may be explained by the decreased demand at these two locations in 2000.

Another surprising finding is that hypothesis $4\left(q_{a, o n}>q_{a, \text { off }}\right)$ is also confirmed. The average flow rate in the pre-queue transition period is about two percent higher with metering than without metering, when averaged across all studied bottlenecks. The difference is statistically significant at level 0.01 . This implies that at the same demand level, breakdown at a bottleneck is less likely to occur if ramp meters control the freeway. Persaud et al. (2001) propose a probabilistic ramp control logic to maximize the expected benefits based on the observed breakdown probability distribution. If the breakdown probability function itself shifts as ramp meters are deployed, one should base metering rates on the distribution estimated from metering-on data. Future studies can estimate and compare the breakdown probability distributions with data collected under both control scenarios, and summarize the implications for the design of efficient and reliable ramp control strategies.

The assumption of constant QDFs at the same bottleneck regardless of metering status has been made in numerous theoretical and practical ramp metering studies. Many believe that once a bottleneck is activated, ramp metering would no longer make a difference and emphasize solely its role of delaying breakdown onset. However, our results suggest that this assumption is not true. Across all studied bottlenecks, $q_{d}$ is more than three percent higher with metering than without metering. The null hypothesis that the two are equal is rejected at level 0.01 . At two locations, $q_{d, o n}$ is as much as nine percent higher than $q_{d, \text { off }}$ (24: a weaving section; and 26: a busy entrance ramp with combined horizontal and vertical curves). A possible explanation is that when an activated bottleneck is not metered, a platoon of merging and/or diverging vehicles may cause intensive short-term lane-changing maneuvers on freeway mainline sections, which may lead to further speed drops and thus $q_{d}$ drops. Another explanation, suggested by an anonymous reviewer, is that QDF at weaving bottlenecks (and bottlenecks with high merging on-ramp flow) is a function of weaving flow determined by freeway OD patterns. The weaving patterns likely changed after the meters were turned off because meters explicitly favor mainline traffic at the expense of ramp traffic. Future studies should examine the causes of lower $q_{d}$ without metering with higher quality data. Cassidy

Table 3

Impacts of traffic growth on bottleneck flow characteristics.

\begin{tabular}{|c|c|c|c|c|c|c|c|c|c|c|c|c|c|}
\hline \multirow[t]{2}{*}{ Id } & \multicolumn{2}{|c|}{ Study period (days) } & \multicolumn{2}{|c|}{ Breakdowns per day } & \multicolumn{3}{|c|}{$q_{d}(\mathrm{veh} / \mathrm{h})$} & \multicolumn{3}{|c|}{$T$ (hh:mm/day) } & \multicolumn{3}{|c|}{$q_{a}(\mathrm{veh} / \mathrm{h})$} \\
\hline & 1999 & 2000 & 1999 & 2000 & 1999 & 2000 & $\Delta(\%)$ & 1999 & 2000 & $\Delta(\%)$ & 1999 & 2000 & $\Delta(\%)$ \\
\hline 1 & 27 & 23 & 3.0 & 1.7 & 4343 & 4378 & 1 & $0: 22$ & $0: 37$ & 68 & 4404 & 4405 & 0 \\
\hline 2 & 28 & 23 & 0.3 & 0.6 & 3958 & 3985 & 1 & $2: 43$ & $2: 41$ & -1 & 4307 & 4330 & 1 \\
\hline 4 & 28 & 25 & 0.1 & 1.5 & 5862 & 6002 & 2 & $2: 39$ & $2: 03$ & -23 & 6461 & 6475 & 0 \\
\hline 6 & 27 & 25 & 1.0 & 0.1 & 3950 & 3608 & -9 & $0: 36$ & $0: 52$ & 44 & 4045 & 4073 & 1 \\
\hline 7 & 28 & 24 & 0.2 & 0 & 4121 & NA & NA & $0: 46$ & $1: 00$ & 30 & 4235 & 4293 & 1 \\
\hline 9 & 28 & 24 & 0.1 & 0.2 & 9095 & 8626 & -5 & $1: 53$ & $1: 17$ & -32 & 9580 & 9525 & -1 \\
\hline 14 & 27 & 23 & 2.5 & 1.2 & 5309 & 5333 & 0 & $0: 50$ & $0: 55$ & 10 & 5446 & 5420 & 0 \\
\hline 15 & 28 & 25 & 0.9 & 0.1 & 4103 & 4043 & -1 & $1: 08$ & $1: 34$ & 38 & 4252 & 4276 & 1 \\
\hline 16 & 25 & 24 & 0.2 & 0.1 & 3862 & 3712 & -4 & $1: 39$ & $1: 14$ & -25 & 4154 & 4081 & -2 \\
\hline 23 & 27 & 23 & 2.7 & 1.8 & 6235 & 6327 & 1 & $1: 08$ & $1: 34$ & 38 & 6522 & 6601 & 1 \\
\hline 26 & 28 & 24 & 0.1 & 0.1 & 6012 & 6051 & 1 & $1: 43$ & $1: 23$ & -19 & 6221 & 6204 & 0 \\
\hline Ave. & 27 & 24 & 1.0 & 0.7 & 5273 & 5207 & -1 & $1: 24$ & $1: 22$ & -2 & 5421 & 5426 & 0 \\
\hline
\end{tabular}

Note: "2000" refers to the seven-week period immediately before the shut down experiment (August 28-October 13, 2000). "1999" refers to the sevenweek period in 1999 (August 30-October 15, 1999) that corresponds to the "2000" period. The purpose of this analysis is to test whether the changes revealed in Table 1 are simply due to annual traffic growth, and that hypothesis is rejected. Ramp metering was on during both periods reported in this table. 
and Rudjanakanoknad (2002) collected arrival times of individual vehicles at a merging bottleneck and found slow-downs even after breakdowns.

Since hypotheses 3-5 are all confirmed by the data, hypothesis 6 is also supported. Ramp metering increases the capacity of freeway bottlenecks in three ways: metering postpones and sometimes eliminates bottleneck activation, it accommodates higher flows during the pre-queue transition period than without metering, and it increases queue discharge flow rates after breakdown. Various parameters defined in the Minnesota zonal ramp control strategy, such as bottleneck flow thresholds, and even the algorithm itself are not necessarily optimal. Therefore, this study may have underestimated the capability of ramp meters. In order to explore the full potential of ramp meters in improving bottleneck capacity, more elaborate experiments in which metering rates can be changed over time are required.

It should be noted that ramp meters increased bottleneck capacity in 1999, when the overall system demand is lower than the metering-off period in 2000 due to annual traffic growth. Thus, it is reasonable to suspect that the observed capacity improvements with metering are simply due to lower demand. To explicitly control for annual traffic growth, all the analyses were repeated for the seven weeks (August 28-October 13, 2000) preceding the metering-off period. This reveals if there are similar results comparing to the corresponding seven new weeks in 1999 (August 30-October 15, 1999). If hypotheses 3-5 are also supported by this new data set, traffic growth clearly plays a role in capacity increases. If they are all rejected, the observed capacity increase at bottlenecks should be mostly attributed to ramp meters. The analysis on the new data set can only be performed at 11 of all 27 study sites for two reasons. First, there were not enough breakdown observations at some locations during the two new seven-week periods because ramp meters were present in both periods. The other reason is related to detector failure. Many corrupt detectors were repaired or replaced immediately before the shut down experiment, which means some bottlenecks with valid traffic data during the metering-off period did not meet the same data requirements during the new seven-week period in 2000. Results of this additional analysis are summarized in Table 3 . Hypotheses 3-5 are all rejected, and no statistically significant differences exist between $q_{d}, T$, or $q_{a}$ during the two new seven-week periods. Therefore the hypothesis that the observed capacity increase is simply due to traffic growth (or decline) is rejected.

An aggregated approach is followed and results from 27 bottlenecks are combined together in this research to study the relationships between ramp metering and bottleneck capacity. However, insights might be lost in the aggregation and there might be advantages to giving more individualized attention to bottlenecks. As seen in Table 1, capacity improvement at different sites varies. In Table 4.1, results are reported for each class of bottlenecks in an attempt to attribute this variation to bottleneck types. Considering some bottlenecks belong to multiple categories, we also performed a regression analysis (see results Table 4.2). It seems that ramp metering can most effectively improve bottlenecks that involve weaving sections, entrance ramps, vertical curves and lane drop, while making little difference at bottlenecks that are bridges/tunnels, or have horizontal curves. Future studies may analyze data at individual bottlenecks in greater detail to provide conclusive findings on why queue discharge flows are higher, transition flows higher, and transition periods longer with ramp metering.

Table 4.1

Results: capacity improvement by bottleneck types.

\begin{tabular}{|c|c|c|c|}
\hline Bottleneck type & \% Change in $q_{d}$ & \% Change in $T$ & $\%$ Change in $q_{a}$ \\
\hline Weaving & 3.7 & 5.9 & 4.4 \\
\hline On-ramp & 3.5 & 2.0 & 1.8 \\
\hline Bridge/tunnel & NA & 0.7 & 1.8 \\
\hline Horizontal curve & 1.8 & 0.8 & 1.0 \\
\hline Vertical curve & 6.2 & 2.1 & 2.3 \\
\hline Lane drop & 3.7 & 0.8 & 3.2 \\
\hline
\end{tabular}

Table 4.2

Regression results: capacity improvement as a function of bottleneck types.

\begin{tabular}{|c|c|c|c|}
\hline \multirow[t]{2}{*}{ Bottleneck type } & \multicolumn{3}{|l|}{ Dependent variables } \\
\hline & $\%$ Change in $q_{d} n=13, R^{2}=0.7$ & $\%$ Change in $T / 100 n=27, R^{2}=0.40$ & $\%$ Change in $q_{a} n=27, R^{2}=0.71$ \\
\hline Weaving & $4.8^{*}$ & $6.7^{* * *}$ & $5.3^{* * *}$ \\
\hline On-ramp & 6.0 & $7.5^{*}$ & $7.5^{* * *}$ \\
\hline Bridge/tunnel & Dropped & -0.72 & $0.5^{* *}$ \\
\hline Horizontal curve & -4.2 & -0.57 & -0.02 \\
\hline Vertical curve & $8.1^{*}$ & 1.94 & $1.7^{*}$ \\
\hline Lane drop & 7.4 & 7.5 & $10.1^{* * *}$ \\
\hline Constant & -3.7 & -6.6 & $-6.9^{* * *}$ \\
\hline
\end{tabular}

Notes: Numbers shown are regression coefficients; all bottleneck types are treated as $(0,1)$ dummy variables.

* Statistically significant at level 0.1 .

** Statistically significant at level 0.05 .

*** Statistically significant at level 0.01 . 
A large number of flow measurements are recorded at the studied bottlenecks, which enables us to examine the distributions of transition flows and discharge flows per interval, per breakdown, and per peak period. The characteristics of these distributions have important bearing on the definition of freeway capacity. A detailed analysis of this issue is, however, beyond the scope of this paper; interested readers are referred to Zhang and Levinson (2004b).

\section{Conclusions}

Traffic flow characteristics at 27 active freeway bottlenecks in the Twin Cities are studied for seven weeks without ramp metering and seven weeks with ramp metering. A series of hypotheses regarding the relationship between ramp metering and the capacity of active bottlenecks are developed and tested against empirical traffic data. The results demonstrate with strong evidence that ramp metering can increase bottleneck capacity. It achieves that by:

(1) Postponing and sometimes eliminating bottleneck activation - the average duration of the pre-queue transition period across all studied bottlenecks is $73 \%$ longer with ramp metering than without;

(2) accommodating higher flows during the pre-queue transition period than without metering - the average flow rate during the transition period is $2 \%$ higher with metering than without (with a $2 \%$ standard deviation); and

(3) increasing queue discharge flow rates after breakdown - the average queue discharge flow rate is $3 \%$ higher with metering than without (with a $3 \%$ standard deviation).

Therefore, ramp meters can reduce freeway delays through not only increased capacity at segments upstream of bottlenecks (type I capacity increase), but also increased capacity at bottlenecks themselves (type II capacity increase). Previously, ramp metering is considered to be effective only when freeway traffic is successfully restricted in uncongested states. The existence of type II capacity increase suggests there are benefits to meter entrance ramps even after breakdown has occurred. This study focuses on the impacts of ramp metering on freeway bottleneck capacity. The causes of such impacts should be more thoroughly examined by future studies, so that the findings can provide more guidance to the development of ramp control strategies. It should also be noted that both types of capacity increases on the freeway mainline are at the expense of degraded conditions at the on-ramps and possibly arterial network. Therefore, without more comprehensive system-wide analysis, the findings of this paper, though in favor of ramp metering, do not necessarily justify its deployment.

Type I and type II capacity increases and metering rates are highly correlated. The premise of type I capacity increase is that the bottlenecks are not activated or the physical queues upstream of bottlenecks do not block exit ramps. The tradeoff here is that metering rates should not be too low such that demand is overly restricted, and should not be too high such that queues form. The traditional metering goal of keeping the flow at a bottleneck strictly below its capacity should provide an optimum solution to this tradeoff. However, this goal may not adequately exploit the correlation between the type II capacity increase and metering rates since an always free-flowing freeway, which dictates low control threshold values (optimal volume or density), is not the best solution. In that case, the ability of ramp meters to postpone bottleneck activation and to increase queue discharge flow rates is wasted. Somewhat more aggressive threshold values may improve the overall effectiveness of ramp metering. To maximize the type II capacity increase, a set of rules associating metering rates and real-time flow conditions (mainline, merging and diverging flows and occupancy, etc.) are required. Improved driver behavior models and careful examination of traffic features at bottlenecks with high-quality data are both promising research approaches. It should also be remembered that type I and type II capacity increases tend to compete with each other when a controlled freeway is uncongested. For that reason, multiple metering logics corresponding to different freeway traffic conditions (maybe before and after breakdown) should be considered. These propositions have a long way to go before shedding light on the practical design of control logic. A direct implication of the findings in this study is that when selecting the threshold flow values for bottlenecks, engineers should not simply rely on the long-run queue discharge rate collected during a metering-off period. A markup of that value estimated locally with empirical study may improve the efficiency of the algorithm.

This study arrives at most findings by combining data from many different bottlenecks. Although the aggregation provides an opportunity to examine distributional effects of some important bottleneck properties, it represents a limitation of this work - additional attention should be paid to the traffic evolution at individual bottlenecks. Diagnostic tools, such as curves of cumulative traffic counts and occupancy (Cassidy and Windover, 1995), should be considered to address this limitation.

Finally, it should be noted that the results presented in this paper are all based on traffic data collected in the Twin Cities with the same ramp control strategy. Similar analysis in other metro areas may provide additional insight. Also after the shut down experiment in 2000, a new algorithm with less restriction on on-ramp inflows and more equity considerations has been deployed in the Twin Cities, which provides an opportunity to empirically test the potential causes of increased bottleneck capacity.

\section{Acknowledgements}

The authors want to thank the anonymous reviewers for their constructive comments. 


\section{References}

Banks, J.H., 1990. Flow processes at a freeway bottleneck. Transportation Research Record 1287, 20-28.

Banks, J.H., 1991a. The two-capacity phenomenon: some theoretical issues. Transportation Research Record 1320, $234-241$.

Banks, J.H., 1991b. Two-capacity phenomenon at freeway bottlenecks: a basis for ramp metering? Transportation Research Record $1320,83-90$.

Bogenberger, K., May, A.D., 1999. Advanced coordinated traffic responsive ramp metering strategies. California PATH Working Paper, UCB-ITS-PWP-99-19. Cambridge Systematics, 2001. Mn/DOT Ramp Metering Study Final Report. Prepared for the Minnesota Department of Transportation. <http:// www.dot.state.mn.us/rampmeterstudy/>.

Cassidy, M., 2003. Freeway on-ramp metering, delay savings and the diverge bottleneck. Paper presented at the 82nd Transportation Research Board Annual Meeting, January 12-16, Washington, DC.

Cassidy, M.J., Bertini, R.L., 1999. Some traffic features at freeway bottlenecks. Transportation Research 33B, 25-42.

Cassidy, M.J., Mauch, M., 2001. An observed traffic pattern in long freeway queues. Transportation Research 35A, 143-156.

Cassidy, M.J., Rudjanakanoknad, J., 2002. Study of traffic at a freeway merge and roles for ramp metering. Final report, California PATH, University of California at Berkeley.

Cassidy, M.J., Windover, J.R., 1995. Methodology for assessing the dynamics of freeway traffic flow. Transportation Research Record 1484, 73-79.

Chen, L., May, A.D., 1987. Traffic detector errors and diagnostics. Transportation Research Record 1132, 82-93.

Daganzo, C.F., 1997. Fundamentals of Transportation and Traffic Operations, vols. 133-135. Elsevier Science, Inc., New York. p. 259.

Hall, F., Agyemang-Duah, K., 1991. Freeway capacity drop and the definition of capacity. Transportation Research Record 1320, 91-98.

Jacobson, L.N., Nihan, N.L., Bender, J.D., 1990. Detecting erroneous loop detector data in a freeway traffic management system. Transportation Research Record 1287, 151-166.

Koshi, M., Kuwahara, M., Akahane, H., 1992. Capacity of sags and tunnels on Japanese motorways. ITE Journal 1992, 17-23.

Kotsialos, A., Papageogiou, M., Mangeas, M., Haj-Salem, H., 2002. Coordinated and integrated control of motorway networks via non-linear optimal control. Transportation Research 10C, 65-84.

Levinson, D., Sheikh, A., 2002. Traffic equilibration: the case of the Twin Cities ramp meter shut off. In: Proceedings of International Conference on Traffic and Transportation Studies, Guilin, China, July 2002, pp. 580-587.

Levinson, D., Zhang, L., 2006. Ramp meters on trial: evidence from the Twin Cities metering holidays. Transportation Research 40A (10), 810-828.

May, A.D., 1964. Experimentation with manual and automatic ramp control. Highway Research Record 59, 9-38.

Minnesota Department of Transportation, 1998. Ramp metering by zone - The Minnesota algorithm. Metro Traffic Management Center Report.

Minnesota Department of Transportation, 2000. MnDOT Traffic Management Center all detector report: October 04, 2000. Report available in the MnDOT library.

Newman, L., 1961. Study of traffic capacity and delay at the merge of the North Sacramento and Elvas freeways. Final Report, California Division of Highways, USA

Newman, L., Dunnet, A., Meirs, J., 1969. Freeway ramp control - what it can and cannot do. Traffic Engineering 19 (7), 14-25.

Persaud, B.N., 1986. Study of a freeway bottleneck to explore some unresolved traffic flow issues. PhD Dissertation, University of Toronto, Canada.

Persaud, B.N., Hurdle, V.F., 1991. Freeway capacity: definition and measurement issues. In: Proceedings of the International Symposium of Highway Capacity, A.A. Balkema Press, Germany, pp. 289-307.

Persaud, B.N., Yagar, S., Brownlee, R., 1998. Exploration of the breakdown phenomenon in freeway traffic. Transportation Research Record 1634, 64-69.

Persaud, B., Yagar, S., Tsui, D., Look, H., 2001. Breakdown-related capacity for freeway with ramp metering. Transportation Research Record 1748, 110-115. Shapiro, S.S., Wilk, M.B., 1965. An analysis of variance test for normality (complete samples). Biometrika 52 (3\&4), 591-611.

TDRL Data Center, 2004. TDRL Utility Software Download Page. <http://www.d.umn.edu/ tkwon/TDRLSoftware/Download.html> (accessed 12.10.04).

Windover, J.R., Cassidy, M.J., 2001. Some observed details of freeway traffic evolution. Transportation Research 35A, 881-894.

Zhang, L., 2007. Individual and system-level traffic diversion impacts of freeway operations. Journal of the Transportation Research Board $2012,20-29$.

Zhang, L., Levinson, D., 2002. Estimation of the demand responses to ramp metering. In: Proceedings of the 3rd International Conference on Traffic and Transportation Studies, July 23-25, Guilin, China, vol. 1, pp. 674-681.

Zhang, L., Levinson, D., 2004a. Optimal freeway ramp control without origin-destination Information. Transportation Research Part B: Methodological 38 (10), 869-887.

Zhang, L., Levinson, D., 2004b. Some properties of flows at freeway bottlenecks. Journal of the Transportation Research Board 1883, 122-131. 